\title{
Higgs inflation and cosmological electroweak phase transition with $N$ scalars in the post-Higgs era
}

\author{
Wei Cheng \\ Department of Physics, Chongqing University, Chongqing 401331, China \\ Ligong Bian* \\ Department of Physics, Chongqing University, Chongqing 401331, China \\ and Department of Physics, Chung-Ang University, Seoul 06974, Korea
}

(Received 18 November 2018; revised manuscript received 22 January 2019; published 26 February 2019)

\begin{abstract}
We study inflation and cosmological electroweak phase transitions utilizing the standard model augmented by $N$ scalars respecting a global $O(N)$ symmetry. We observe that the representation of the global symmetry is restricted by the inflationary observables and the condition of a strongly first-order electroweak phase transition. Theoretical constraints including the stability, perturbativity, and unitarity are used to bound the model parameter space. The electroweak precision observables and Higgs precision limit the representation of the symmetry. We evaluate the possibility to simultaneously address the inflation and the dark matter after considering the experimental constraints from the future leptonic colliders. When the $O(N)$ symmetry respected by the $N$ scalar is spontaneously broken to the $O(N-1)$ symmetry, both the one-step and two-step SFOEWPT can occur within the inflation viable parameter regions, which will be tested by the future CEPC, ILC, and FCC-ee. The relation between the number of Goldstones and the SFOEWPT condition depends on phase transition patterns. The situations of Goldstone faking neutrinos and contributions to dark radiation are investigated.
\end{abstract}

DOI: 10.1103/PhysRevD.99.035038

\section{INTRODUCTION}

To our knowledge, the standard model of particle physics $(\mathrm{SM})$ is incapable of explaining the three longstanding problems of particle physics and cosmology, i.e., the horizon, flatness, and monopole problems of the Universe, the baryon asymmetry of the Universe (BAU), and the existence of dark matter. The cosmic inflation [1-3] solves the first one successfully. The primordial density fluctuations generated during inflation can explain the formation of the large-scale structure of the Universe observed by CMB [4]. The inflation scenario is attractive when the inflaton field can play an important role in particle physics. A fascinating scenario is the Higgs inflation [5-10], where the inflaton is the SM Higgs being observed by LHC [14,15]. There is a lot of debate on whether the Higgs inflation suffers from the unitarity problem at high scales around $\sim \mathcal{O}\left(10^{13}\right) \mathrm{GeV}$ [16-27], which is beyond the scope of this paper. Among various mechanisms to explain the BAU, the electroweak

\footnotetext{
*1gbycl@cqu.edu.cn
}

Published by the American Physical Society under the terms of the Creative Commons Attribution 4.0 International license. Further distribution of this work must maintain attribution to the author(s) and the published article's title, journal citation, and DOI. Funded by SCOAP. baryogenesis mechanism (EWBG) raises people's interest due to the two essential ingredients that are able to be tested in experiments. A strongly first-order electroweak phase transition (SFOEWPT) as an essential ingredient usually requires the extension of the Higgs sector of the SM [29,30], and the modified scalar potential could be detected at hadronic and leptonic colliders [31]. The additional $C P$ violation, as another essential ingredient for the EWBG, can be probed indirectly with the electric dipole moment experiments. The $C P$ violation study is beyond the scope of this paper, though it may affect the phase transition.

To realize a SFOEWPT, one simplest and extensively studied approach is extending the SM with an additional real singlet scalar [32-38] or complex singlet scalar [39,40] through the Higgs portal. For the Higgs inflation with assistance of singlet scalars utilizing Higgs-portal interactions, we refer to Refs. [41-44]. For the Higgs inflation in the Higgs-portal scenarios, the typical quartic scalar couplings are required to be around $\sim \mathcal{O}\left(10^{-1}\right)[41,42,45]$. To obtain a one-step type SFOEWPT, a relatively large Higgs portal quartic coupling is required [46], which might lead to an unexpected theoretical problem, i.e., breaking the perturbativity, and unitarity at high scale. Therefore, largeness of the quartic couplings cannot accommodate the successful inflation. For the previous attempts to connect cosmic inflation and electroweak phase transition (EWPT) in this case, we refer to Refs. $[45,47,48]$. 
The straightforward approach to ameliorate the situation can be extending the SM by singlet scalars that respect $O(N)$ symmetry, then the one-step SFOEWPT can be realized with a lower magnitude of the Higgs-portal interaction $|H|^{2} S_{i} S_{i}(i=1, \ldots, N)$ [49-52]. Previous studies of $N$ scalars with an exact $O(N)$ symmetry suggest that a one-step SFOEWPT can be realized with a relatively large $\mathrm{N}$, which results in detectable gravitational wave signals with typical frequency of $\sim \mathcal{O}\left(10^{-3}-10^{-1}\right) \mathrm{Hz}$ [50-52] and a substantial triple Higgs coupling deviation to be probed by the future colliders [51,52]. Therefore, we introduce an additional $N$ hidden scalar that respects the $\mathcal{O}(N)$ symmetry and investigate the possibility to accommodate inflation together with a SFOEWPT. Here, the additional $N$ hidden scalars might also alleviate the hierarchy problem through positive contributions to radiative corrections of the Higgs boson mass and therefore satisfy the Veltman conditions [53-55]. As an additional benefit, the scalars can saturate the DM candidate. The previous studies of Ref. [50] indicate that a one-step SFOEWPT cannot be addressed together with a correct DM relic density unless the quartic couplings of $|H|^{2} S_{i} S_{i}$ and masses of $S_{i}$ are nonuniversal [56]. In this work, we study both one-step and two-step EWPT, and evaluate the DM together with the inflation explanation. Our study shows that the electroweak precision observable (EWPOs) constraints invalidate the inflation explanation when $N>4$ for the $\mathcal{O}(N)$ scalars that shout down the window to accommodate a SFOEWPT. We further explore the scenario wherein the $\mathcal{O}(N)$ symmetry is spontaneously broken to the $\mathcal{O}(N-1)$ symmetry. There are $N-1$ Goldstones that can fake the effective neutrinos. The possibility of Goldstones contributing to dark radiation provided they gain masses from nonrenormalizable gravity effects will be estimated. Our results demonstrate that, after considering the theoretical constraints and the current Higgs precisions, the inflation explanation and a SFOEWPT can be reached in certain parameter spaces for both one- and twostep phase transitions.

The paper is organized as follows: In Sec. II, we introduce the model including the case of the scalars respecting the $O(N)$ symmetry and the scenario where the $\mathcal{O}(N)$ is spontaneously broke to the $\mathcal{O}(N-1)$, the relevant theoretical constraints and the Higgs precision constraints are explored. Cosmological implications to be studied including inflation, electroweak phase transition, dark matter and dark radiation are given in Sec. III. The numerical results for both the $\mathcal{O}(N)$ and $\mathcal{O}(N-1)$ scenarios are presented in Sec. IV. We conclude with Sec. V.

\section{THE MODELS}

In this work, we study two scenarios of $N$-singlet scalar extended SM. In the case of the $N$-singlet scalars $(S)$ with $O(N)$ symmetry, the $O(N)$ symmetry might break at finite temperature and restore at zero temperature.
Another scenario is that the $O(N)$ is spontaneously broken to $O(N-1)$ at zero temperature; we use "s" rather than " $S$ " to differentiate it from the $O(N)$ scenario.

For the $O(N)$ scenario, the zero temperature tree-level potential is given by

$$
\begin{aligned}
V_{0}(H, S)= & -\mu_{h}^{2} H^{\dagger} H+\lambda_{h}\left|H^{\dagger} H\right|^{2}+\frac{\mu_{s}^{2}}{2} S_{i} S_{i}+\frac{\lambda_{s}}{4}\left(S_{i} S_{i}\right)^{2} \\
& +\frac{1}{2} \lambda_{h s}|H|^{2} S_{i} S_{i},
\end{aligned}
$$

with $H^{T}=\left(G^{+},\left(v+h+i G^{0}\right) / \sqrt{2}\right)$. After the spontaneous symmetry breaking of the electroweak symmetry, the mass term of $S_{i}$ is given as $m_{S_{i}}^{2}=\mu_{s}^{2}+\lambda_{h s} v^{2} / 2$. For the $O(N \rightarrow N-1)$ scenario, the minimization conditions of the potential can be obtained when EW symmetry is broken and the $O(N)$ being broke along the direction of $s$, with other directions being $s_{i}(i=1, \ldots, N-1)$ [58],

$$
\left.\frac{d V_{0}(h, s, A)}{d h}\right|_{h=v}=0,\left.\quad \frac{d V_{0}(h, s, A)}{d s}\right|_{s=v_{s}}=0,
$$

which give rise to $\mu_{h}^{2}=\lambda_{h} v^{2}+\lambda_{h s} v_{s}^{2} / 2, \mu_{s}^{2}=-\left(\lambda_{h s} v^{2} / 2+\right.$ $\left.\lambda_{s} v_{s}^{2}\right)$. The mass matrix is given by

$$
\mathcal{M}^{2}=\left(\begin{array}{cc}
2 v^{2} \lambda_{h} & v v_{s} \lambda_{h s} \\
v v_{s} \lambda_{h s} & 2 v_{s}^{2} \lambda_{s}
\end{array}\right)
$$

In order to diagonalize the mass matrix, we introduce the rotation matrix $R=((\cos \theta, \sin \theta),(-\sin \theta, \cos \theta))$ with $\tan 2 \theta=-\lambda_{h s} v v_{s} /\left(\lambda_{h} v^{2}-\lambda_{s} v_{s}^{2}\right)$ to relate the mass basis and field basis,

$$
\left(\begin{array}{l}
h \\
s
\end{array}\right)=\left(\begin{array}{cc}
\cos \theta & \sin \theta \\
-\sin \theta & \cos \theta
\end{array}\right)\left(\begin{array}{l}
h_{1} \\
h_{2}
\end{array}\right) .
$$

The mass squared eigenvalues are

$$
m_{h_{1}, h_{2}}^{2}=\lambda_{h} v^{2}+\lambda_{s} v_{s}^{2} \mp \frac{\lambda_{s} v_{s}^{2}-\lambda_{h} v^{2}}{\cos 2 \theta} .
$$

Identify the $h_{1}$ being the $126 \mathrm{GeV}$ SM-like Higgs boson, and requiring the $h_{2}$ is dominated by $s$ set $\cos \theta>1 / \sqrt{2}$. Here, we note that the situation of $m_{h_{2}}>m_{h_{1}}$ and $|\theta|<\pi / 4$ correspond to $\lambda_{s} v_{s}^{2}>\lambda_{h} v^{2}$. The quartic couplings can be expressed as functions of the Higgs masses, $v, v_{s}$ and the mixing angle $\theta$,

$$
\begin{aligned}
& \lambda_{h}=\frac{m_{h_{2}}^{2} \sin ^{2} \theta+m_{h_{1}}^{2} \cos ^{2} \theta}{2 v^{2}}, \\
& \lambda_{s}=\frac{m_{h_{2}}^{2} \cos ^{2} \theta+m_{h_{1}}^{2} \sin ^{2} \theta}{2 v_{s}^{2}},
\end{aligned}
$$




$$
\lambda_{h s}=\frac{\left(m_{h_{2}}^{2}-m_{h_{1}}^{2}\right) \sin 2 \theta}{2 v v_{s}} .
$$

We note that in our parameterization, the positiveness of the squared Higgs mass eigenvalues [given in Eq. (5)] is justified when the determinant of the Hessian matrix [Eq. (3)] is positive, which leads to $4 \lambda_{h} \lambda_{s}-\lambda_{h s}^{2}>0$, and results in a condition of $m_{h_{2}}^{2} m_{h_{1}}^{2} /\left(v^{2} v_{s}^{2}\right)>0$ after considering the Eq. (8).

The number of scalars $(N)$ or Goldstones $(N-1)$, and scalar quartic couplings $\lambda_{s, h s, h}$ in the interaction basis for the $O(N)$ and $O(N \rightarrow N-1)$ scenarios will be constrained by the perturbativity and unitarity, stability, and Higgs precisions, as well as EWPOs. The parameter spaces will be further restricted by the inflationary observables and the condition of SFOEWPT, which will be studied in the following sections. As will be studied in the following sections, in both the $O(N)$ and the $O(N \rightarrow N-1)$ scenarios, the number of $N$ will be bounded by the condition of the slow-roll inflation and a SFOEWPT. For the $O(N \rightarrow N-1)$, it means the number of Goldstones is bounded. As will be shown later, for the light extra Higgs mass, the dark radiation set bounds on the number of Goldstones in the $O(N \rightarrow$ $N-1)$ scenario.

\section{A. Theoretical constraints}

Firstly, due to the additive property of the scalar quartic couplings contribution to the beta functions (see Appendix A), one need to aware the possible perturbativity problem at high scale when one performs the inflation analysis. We impose the following conditions to preserve the perturbativity,

$$
\left|\lambda_{h}\right|<1, \quad\left|\lambda_{s}\right|<\sqrt{4 \pi}, \quad\left|\lambda_{h s}\right|<\sqrt{4 \pi} .
$$

The perturbative unitarity condition is obtained by requiring that the absolute value of the s-wave $2 \rightarrow 2$ scattering amplitudes among the longitudinal gauge bosons and scalars is smaller than $1 / 2$, which sets bounds on scalar quartic coupling of the tree-level potential for $O(N)$ and $O(N \rightarrow N-1)$ scenarios as follows [52],

$\frac{1}{32 \pi}\left(3 \lambda+(N+2) \lambda_{s}+\sqrt{\left(3 \lambda-(N+2) \lambda_{s}\right)^{2}+4 N \lambda_{h s}^{2}}\right)<\frac{1}{2}$.

To prevent the unbounded from below the scalar potential, the vacuum stability conditions should be satisfied,

$\lambda_{h}>0, \quad \lambda_{s}>0, \quad \lambda_{h s}>0 \quad$ or $\quad \lambda_{h s}>-2 \sqrt{\lambda_{h} \lambda_{s}}$.

Here, for completeness, the last condition include both the scenarios of $\lambda_{h s}>0$ and $\lambda_{h s}<0$. The third equation of

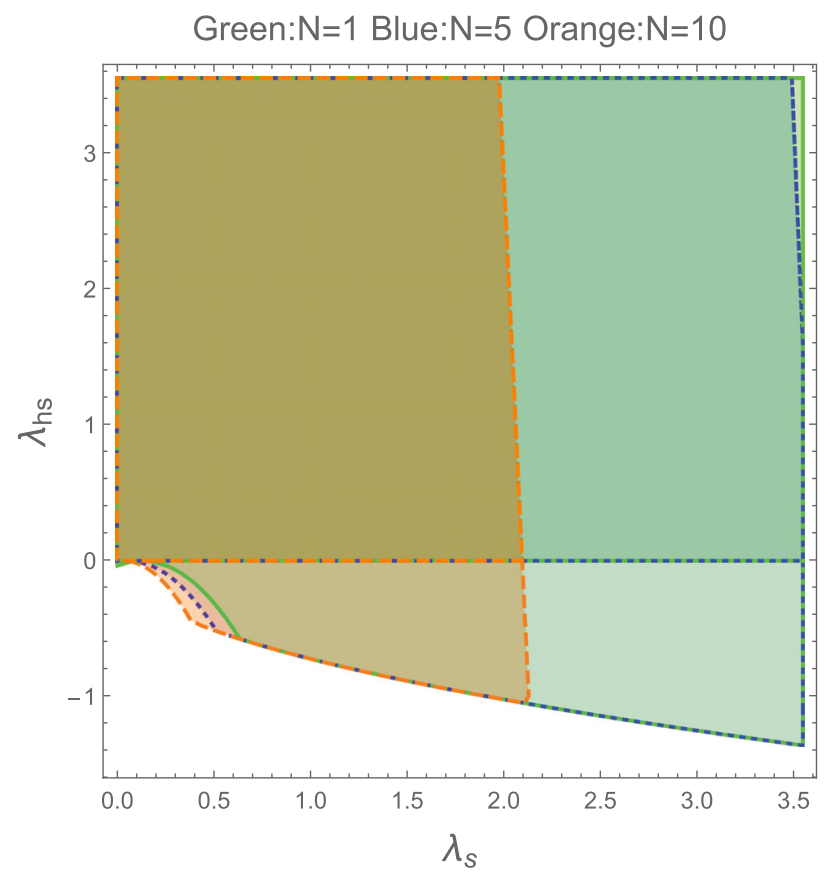

FIG. 1. Parameters regions allowed by Perturbativity + Unitarity + Stability in the $O(N)$ scenario at EW scale. Both the $\lambda_{h s}>0$ and $\lambda_{h s}>-2 \sqrt{\lambda_{h} \lambda_{s}}$ are shown.

Eq. (8) indicates that the scenario of $\lambda_{h s}>0$ corresponds to $m_{h_{2}}>m_{h_{1}}$ in the parameter region of $0<\theta<\pi / 4$.

A simple analysis of these theoretical limits on the scalar quartic couplings at the electroweak scale is given in Figs. 1 and 2 for $O(N)$ and $O(N \rightarrow N-1)$ scenarios. The perturbativity roughly sets the upper limit of $\lambda_{h s, s}$, the shape of the boundary is set by the unitarity bounds. The lower bound of the quartic couplings $\lambda_{h s, s}$ is given by the stability conditions where more parameter spaces are allowed by $\lambda_{h s}>-2 \sqrt{\lambda_{h} \lambda_{s}}$ in comparison with $\lambda_{h s}>0$. In the Fig. 2 , it's converted to the bounds on the $m_{h_{2}}$ and $v_{s}$ correspondingly.

For the study of inflation and EWPT, we implement three conditions of the perturbativity, unitarity, and the stability of the inflationary potential from the electroweak (EW) scale to Planck scale, which are evaluated with the renormalization group equations list in Appendix A.

\section{B. Higgs precisions}

Integrating out the heavy scalar fields results in the dimension-six operators,

$$
\mathcal{L} \supset \frac{c_{H}}{\Lambda^{2}} \mathcal{O}_{H}+\frac{c_{6}}{\Lambda^{2}} \mathcal{O}_{6}
$$

with $O_{H} \equiv \frac{1}{2}\left(\partial\left|H^{\dagger} H\right|\right)^{2}$ and $\mathcal{O}_{6} \equiv\left|H^{\dagger} H\right|^{3}$. Here, the operator $\mathcal{O}_{H}$ can lead to the universal shift of Higgs couplings by the Higgs field redefinition or Higgs wave-function renormalization. The operator $\mathcal{O}_{6}$ can alleviate the triple Higgs coupling and is crucial for the realization of a SFOEWPT [59]. For the Wilson coefficients generated at tree level, we have $[60,61]$ 

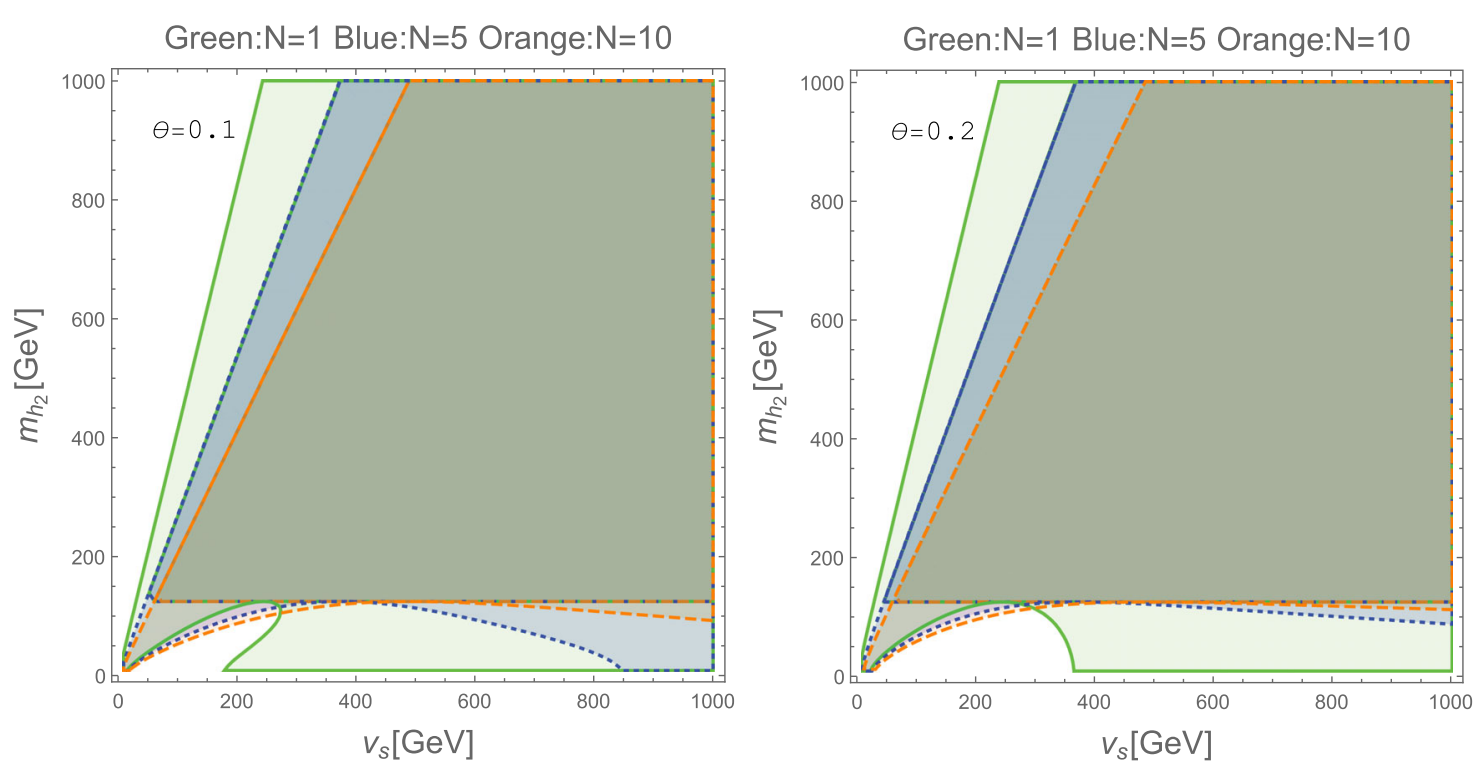

FIG. 2. Parameters regions allowed by Perturbativity + Unitarity + Stability in the $O(N \rightarrow N-1)$ scenario at EW scale.

$$
\begin{gathered}
c_{H}^{N}=N \frac{\lambda_{h s}^{2}}{2 \lambda_{s}}, \quad c_{6}^{N}=0, \\
c_{H}^{N \rightarrow N-1}=\frac{\lambda_{h s}^{2}}{2 \lambda_{s}}, \quad c_{6}^{N \rightarrow N-1}=0,
\end{gathered}
$$

with the $\Lambda \approx \mu_{s}$ for the two scenario. For $O(N)$ case one have $\mu_{s} \approx m_{S_{i}}$, and in the $O(N \rightarrow N-1)$ case $\mu_{s} \approx m_{h_{2}}$ for the small mixing angle limit. The loop-level induced dimensionsix operator Wilson coefficients are

$$
c_{H}^{N}=\frac{N \lambda_{h s}}{48 \pi^{2}}, \quad c_{6}^{N}=-\frac{N \lambda_{h s}^{3}}{48 \pi^{2}}
$$

for the $O(N)$ case. These reduce to

$$
c_{H}^{N \rightarrow N-1}=\frac{\lambda_{h s}}{48 \pi^{2}}, \quad c_{6}^{N \rightarrow N-1}=-\frac{\lambda_{h s}^{3}}{48 \pi^{2}}
$$

for the $O(N \rightarrow N-1)$ scenario. We note that this is the same with the tree-level induced dimension-six operator, where the factor of " $N$ " in $O(N \rightarrow N-1)$ does not appear in Eq. (16) because there is only one heavy scalar and the other $s_{N-1}$ scalars are massless Goldstones. For the quartic coupling $\lambda_{h s} \sim \mathcal{O}\left(10^{-1}\right)$ being required by the inflation, we can safely ignore the loop-level induced operator effects on the Higgs precision. In the small mixing angle limit of the $O(N \rightarrow$ $N-1)$ scenario, we have $1-\cos \theta \approx c_{H}^{N \rightarrow N-1} v^{2} /\left(2 m_{h_{2}}^{2}\right) \approx$ $c_{H}^{N \rightarrow N-1} v^{2} / 2 \mu_{s}^{2}$.

Now, we explore the EWPOs in the two scenarios. The operator $\mathcal{O}_{H}$ induces the operator combinations $O_{W}+O_{B}$ and $O_{T}$ operators through RGE $[60,62]$, which results in the $\mathrm{S}$ and $\mathrm{T}$ parameters

$$
\begin{gathered}
\Delta S=\frac{1}{12} c_{H} \frac{v^{2}}{m_{S\left(h_{2}\right)}^{2}} \log \left(\frac{m_{S\left(h_{2}\right)}^{2}}{m_{W}^{2}}\right), \\
\Delta T=-\frac{3}{16 \pi c_{W}^{2}} c_{H} \frac{v^{2}}{m_{S\left(h_{2}\right)}^{2}} \log \left(\frac{m_{S\left(h_{2}\right)}^{2}}{m_{W}^{2}}\right) .
\end{gathered}
$$

We set bounds on $m_{S\left(h_{2}\right)}$ and the " $N$ " using the electroweak fit in Ref. [63],

$$
S=0.06 \pm 0.09, \quad T=0.10 \pm 0.07,
$$

with the correlation coefficient between the $\mathrm{S}$ and $\mathrm{T}$ parameters being +0.91 . In the case of $O(N \rightarrow N-1)$, the parameter spaces are more strictly constrained by $\mathrm{T}$ parameter rather than $\mathrm{S}$ parameter, which set stringent bounds on the mixing angle and the masses of the heavy Higgs. When the heavy Higgs is not highly decoupled [64], i.e., $m_{h_{2}} \sim m_{h}$, one can obtain the oblique parameter $\mathrm{T}$ following Ref. [65],

$$
\begin{aligned}
T= & -\left(\frac{3}{16 \pi s_{W}^{2}}\right)\left\{\operatorname { c o s } ^ { 2 } \theta \left[\frac{1}{c_{W}^{2}}\left(\frac{m_{h_{1}}^{2}}{m_{h_{1}}^{2}-M_{Z}^{2}}\right) \ln \frac{m_{h_{1}}^{2}}{M_{Z}^{2}}\right.\right. \\
& \left.-\left(\frac{m_{h_{1}}^{2}}{m_{h_{1}}^{2}-M_{W}^{2}}\right) \ln \frac{m_{h_{1}}^{2}}{M_{W}^{2}}\right]+\sin ^{2} \theta\left[\frac{1}{c_{W}^{2}}\left(\frac{m_{h_{2}}^{2}}{m_{h_{2}}^{2}-M_{Z}^{2}}\right)\right. \\
& \left.\left.\times \ln \frac{m_{h_{2}}^{2}}{M_{Z}^{2}}-\left(\frac{m_{h_{2}}^{2}}{m_{h_{2}}^{2}-M_{W}^{2}}\right) \ln \frac{m_{h_{2}}^{2}}{M_{W}^{2}}\right]\right\} .
\end{aligned}
$$

For the $O(N \rightarrow N-1)$ scenario, the mixing angle and the heavy Higgs masses are subjective to the bounds coming from the LHC Higgs data, which limit the mixing angle $\theta$ to be $|\cos \theta| \geq 0.84$ [33]. After including the 
current LHC and High-luminosity LHC Higgs production rates together with the EWPOs, a moderate of $\theta \sim \sqrt{\lambda_{h s}^{2} v^{2} /\left(4 \lambda_{s} m_{h_{2}}^{2}\right)}=0.2$ can be safety [66]. We firstly perform the Higgs fit without including the change of SM Higgs decay width induced by the Goldstone, and then constrain the number of Goldstone bosons $N-1$ with the Higgs invisible decay fit results from [67]: $B_{\mathrm{BSM}}<0.34$ at $95 \% \mathrm{CL}$. For the case of $O(N)$ symmetry being broken to $O(N-1)$ at zero temperature, we have the following Lagrangian to describe the triple scalar interactions,

$$
\mathcal{L} \supset \lambda_{h_{i} h_{j} h_{j}} h_{i} h_{j} h_{j}+\lambda_{h_{i} s_{N-1} s_{N-1}} h_{i} s_{N-1} s_{N-1},
$$

with $h_{i, j}$ denotes $h_{1,2}$ and $N=2, \ldots, N$, the relevant triple scalar couplings are given below,

$$
\lambda_{h_{2} h_{1} h_{1}}=-\frac{m_{h_{1}}^{2}}{2 v v_{s}} \sin (2 \theta)\left(v_{s} \cos \theta+v \sin \theta\right)\left(1+m_{h_{2}}^{2} / 2 m_{h_{1}}^{2}\right),
$$

$$
\begin{gathered}
\lambda_{h_{2} s_{N-1} s_{N-1}}=m_{h_{2}}^{2} \cos \theta /\left(2 v_{s}\right), \\
\lambda_{h_{1} s_{N-1} s_{N-1}}=-m_{h_{1}}^{2} \sin \theta /\left(2 v_{s}\right), \\
\lambda_{h_{1} h_{2} h_{2}}=\lambda_{h_{1} s_{N-1} s_{N-1}} .
\end{gathered}
$$

From which, when the $m_{h_{2}}>m_{h_{1}}$, the decay widths of the SM-like Higgs and the second Higgs are given by

$$
\begin{aligned}
& \Gamma_{h_{2}}^{\mathrm{tot}}= \Gamma_{h_{2}}\left(h_{2} \rightarrow h_{1} h_{1}\right)+\left.\sin ^{2} \theta \Gamma_{h}^{\mathrm{SM}}\right|_{m_{h} \rightarrow m_{h_{2}}} \\
&+(N-1) \Gamma_{h_{2}}\left(h_{2} \rightarrow s_{N-1} s_{N-1}\right) \\
&= \Gamma_{h_{2}}\left(h_{2} \rightarrow h_{1} h_{1}\right)+\left.\sin ^{2} \theta \Gamma_{h}^{\mathrm{SM}}\right|_{m_{h} \rightarrow m_{h_{2}}} \\
&+(N-1) \frac{\lambda_{h_{2} s_{N-1} s_{N-1}}^{2}}{32 \pi m_{h_{2}}} \\
& \Gamma_{h_{1}}^{\mathrm{tot}}=\cos ^{2} \theta \Gamma_{h}^{\mathrm{SM}}+(N-1) \Gamma_{h}\left(h \rightarrow s_{N-1} s_{N-1}\right) \\
&=\cos ^{2} \theta \Gamma_{h}^{\mathrm{SM}}+(N-1) \frac{\lambda_{h_{1} s_{N-1} s_{N-1}}^{2 \pi m_{h_{1}}},}{32 \pi m^{2}}
\end{aligned}
$$

with

$$
\Gamma\left(h_{2} \rightarrow h_{1} h_{1}\right)=\frac{\lambda_{h_{2} h_{1} h_{1}}^{2}}{32 \pi m_{h_{2}}} \sqrt{1-4 m_{h_{1}}^{2} / m_{h_{2}}^{2}} .
$$

For the case in which $m_{h_{1}}>2 m_{h_{2}}$, one need take into account the decay of $h_{1} \rightarrow 2 h_{2}$ with the decay width being given by

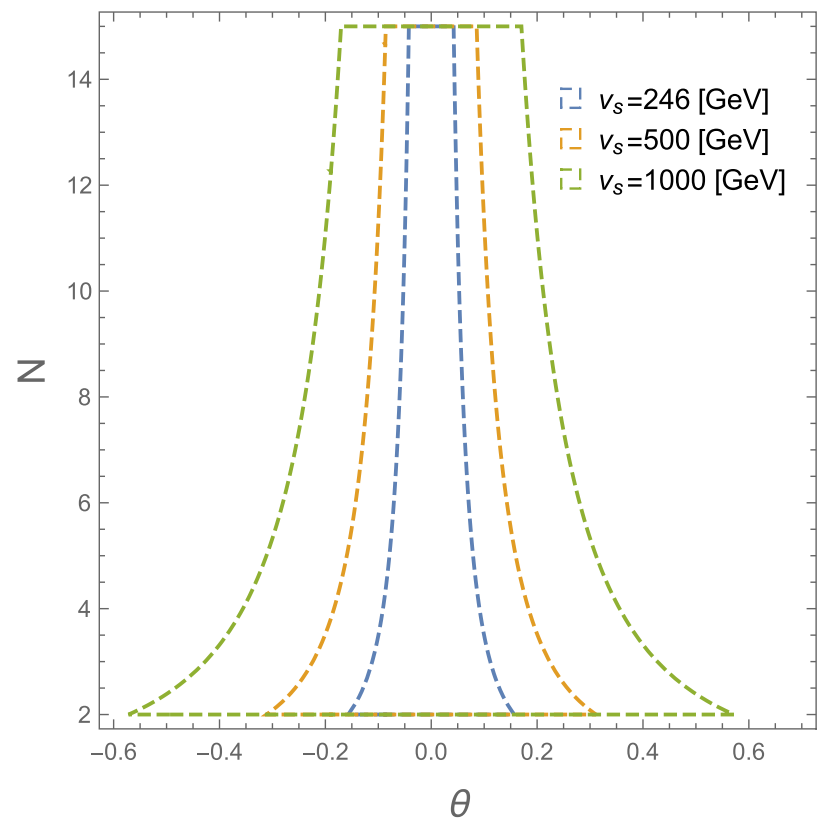

FIG. 3. Invisible decay bounds on $N$ and mixing angle $\theta$ in $O(N \rightarrow N-1)$ model coming from LHC [67].

$$
\Gamma\left(h_{1} \rightarrow h_{2} h_{2}\right)=\frac{\lambda_{h_{1} h_{2} h_{2}}^{2}}{32 \pi m_{h_{1}}} \sqrt{1-4 m_{h_{2}}^{2} / m_{h_{1}}^{2}} .
$$

The invisible decay of SM Higgs can be used to set upper bounds to the number of the Goldstones and the mixing angle $\theta$. At 95\% CL, the LHC (ATLAS + CMS) set $B_{\text {inv }}<$ $34 \%$ [67], see Fig. 3 for the constraints. With the increase of $v_{s}$, more parameter space of $(\theta, N)$ is allowed.

On the other hand, the $\mathcal{O}_{H}$ leads to the modification of the wave function of the Higgs,

$$
\mathcal{L}_{\text {eff }} \supset\left(1+\delta Z_{h}\right) \frac{1}{2}\left(\partial_{\mu} h\right)^{2},
$$

with $\delta Z_{h}=2 v^{2} c_{H} / m_{S\left(h_{2}\right)}^{2}$ for $O(N)(O(N \rightarrow N-1))$ scenarios. Thus, one obtains a universal shift of all Higgs couplings, which therefore induces the correction to the $e^{+} e^{-} \rightarrow h Z$ associated production cross section [62],

$$
\delta \sigma_{Z h}=-2 \frac{v^{2} c_{H}}{m_{S\left(h_{2}\right)}^{2}},
$$

which has been defined as the fractional change in the associated production cross section relative to the SM case. For the $O(N)$ scenario with $m_{S}>m_{h}$, the Higgs wavefunction renormalization shifts the SM-like Higgs couplings to other SM particles by $c_{H}^{N} v^{2} /\left(2 m_{S}^{2}\right) \sim N \lambda_{h s}^{2} v^{2} /$ $\left(2 \lambda_{s} m_{S}^{2}\right)$, which results in the constraint on $c_{H}$ and therefore $N, \lambda_{h s, s}$ from the LHC [66] as well as ILC, CEPC, and FCC-ee [68]. The study of Ref. [68] shows that the CEPC, ILC, and FCC-ee can probe the new physics parameter 


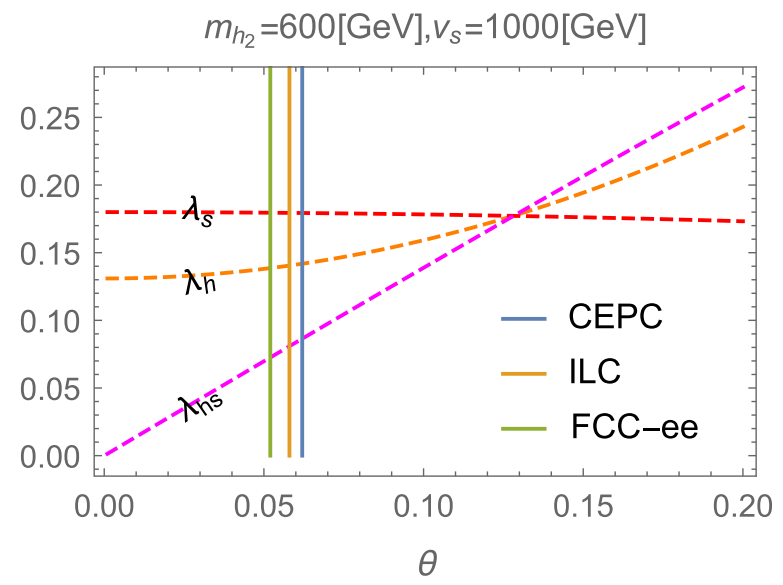

FIG. 4. The projected sensitivity of the mixing angle for $O(N \rightarrow N-1)$ model from lepton colliders.

spaces (through the $e^{+} e^{-} \rightarrow h Z$ process) much better than LHC.

For $N=1$ of the $O(N \rightarrow N-1)$ scenario (which is the Higgs-portal 1-singlet scalar case), the CEPC with luminosity of $5 \mathrm{ab}^{-1}$, ILC with all center-of-mass energies, and FCC-ee with luminosity of $10 \mathrm{ab}^{-1}$ bound the mixing angle $|\sin \theta|$ to be $0.062,0.058$ and 0.052 at $95 \%$ C.L. [68]. We constrain our model parameter spaces with these values, see Fig. 4. These high sensitivity leptonic colliders set a severe bound on the $\lambda_{h s}$, and therefore the stability problem can easily preclude the chance to realize the slow-roll Higgs inflation. For the case of $N \geq 2$ of the $O(N \rightarrow N-1)$ scenario, using the Higgs Strahlung process, the ILC set $B_{\text {inv }}<1 \%$ [69], the FCC-ee set $B_{\text {inv }}<0.5 \%$ [70,71], and CEPC set $0.14 \%$ [72]. Figure 5 indicates that a large mixing angle $\theta$ is allowed for a large $v_{s}$, which corresponds to the heavy Higgs decouple cases. Generally, to make the Higgs inflation feasible, a relatively large mixing angle $\theta$ is required to enlarge the value of $\lambda_{h s}$ and therefore to ensure the vacuum stability. Firstly, the increasing of $\theta$ can lead to the perturbativity problem of $\lambda_{h}$, and thus we need a relatively small $m_{h_{2}}$. Secondly, a large $v_{s}$ leads to a small $\lambda_{h s}$, therefore to avoid the stability problem we need a large $N$. The future $e^{+} e^{-}$colliders constraints give a narrow parameter region of the mixing angle $\theta$. In this super-weak couple scenario the SFOEWPT would not occur due to the $\lambda_{h s}$ is too small, thus one needs a much larger $N$ to amplified the effects of the $N-1$ Goldstones in order to obtain a SFOEWPT. On the other hand, the Goldstones faked effective neutrino situation would be changed a lot due to the decouple conditions allowed parameter spaces can be covered by the bounds from $B_{\text {inv }}$ at ILC, FCC-ee and CEPC as aforementioned.

\section{COSMOLOGICAL IMPLICATIONS}

We first study the cosmic inflation with large-scale fields. With the temperatures of the Universe cooling down the low scale physics come to us: the possibility to obtain a SFOEWPT, and the dark matter physics.

\section{A. The Higgs inflation with $N$-singlet scalars}

The action in the Jordan frame is

$$
\begin{aligned}
S_{J}= & \int d^{4} x \sqrt{-g}\left[-\frac{M_{\mathrm{p}}^{2}}{2} R-\xi_{h}\left(H^{\dagger} H\right) R-\xi_{s} S^{2} R\right. \\
& \left.+\partial_{\mu} H^{\dagger} \partial^{\mu} H+\left(\partial_{\mu} S\right)^{2}-V(H, S)\right],
\end{aligned}
$$

where $M_{\mathrm{p}}$ is the reduced Planck mass, $R$ is the Ricci scalar, $\xi_{\mathrm{h}, \mathrm{S}}$ define the nonminimal coupling of the $h, S$-field. Here, we drop the subscript to simplify the notation as in Ref. [73]. The quantum corrected effective Jordan frame Higgs potential at large field value $(h)$ can be written as

$$
V(h)=\frac{1}{4} \lambda_{h}(\mu) h^{4},
$$
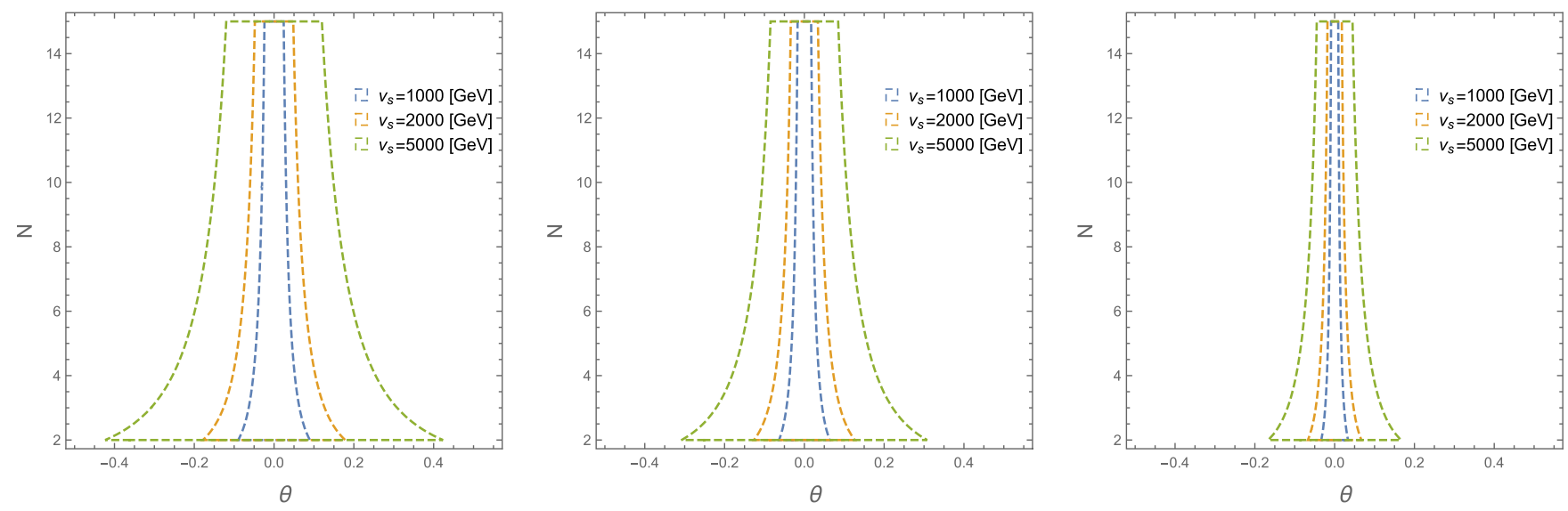

FIG. 5. Higgs invisible decay bounds on $N$ and $\theta$ in $O(N \rightarrow N-1)$ scenario considering the sensitivity of ILC, FCC-ee, and CEPC, respectively, from left to right panels. 
which is evaluated along the higgs axis, where the scale is $\mu \sim \mathcal{O}(h) \approx h$. The potential is the inflationary potential, which will be used to estimate the slow-roll parameters of $\varepsilon$ and $\eta$. We impose quantum corrections to the potential following Refs. [74,75]. After the conformal transformation,

$$
\tilde{g}_{\mu \nu}=\Omega^{2} g_{\mu \nu}, \quad \Omega^{2} \equiv 1+\frac{\xi_{S}}{M_{\mathrm{P}}^{2}} S^{2}+\frac{\xi_{\mathrm{h}} h^{2}}{M_{\mathrm{P}}^{2}} .
$$

and a field redefinition

$\frac{d \chi_{\mathrm{h}}}{d h}=\sqrt{\frac{\Omega^{2}+6 \xi_{\mathrm{h}}^{2} h^{2} / M_{\mathrm{P}}^{2}}{\Omega^{4}}}, \quad \frac{d \chi_{\mathrm{S}}}{d S}=\sqrt{\frac{\Omega^{2}+6 \xi_{S}^{2} S^{2} / M_{\mathrm{P}}^{2}}{\Omega^{4}}}$,

we obtain

$$
\begin{aligned}
S_{E}= & \int d^{4} x \sqrt{-\tilde{g}}\left(-\frac{1}{2} M_{\mathrm{P}}^{2} R+\frac{1}{2} \partial_{\mu} \chi_{\mathrm{h}} \partial^{\mu} \chi_{\mathrm{h}}+\frac{1}{2} \partial_{\mu} \chi_{\mathrm{S}} \partial^{\mu} \chi_{\mathrm{S}}\right. \\
& \left.+A\left(\chi_{\mathrm{S}}, \chi_{\mathrm{h}}\right) \partial_{\mu} \chi_{\mathrm{h}} \partial^{\mu} \chi_{\mathrm{S}}-U\left(\chi_{\mathrm{S}}, \chi_{\mathrm{h}}\right)\right)
\end{aligned}
$$

where $U\left(\chi_{\mathrm{S}}, \chi_{\mathrm{h}}\right)=\Omega^{-4} V\left(S\left(\chi_{\mathrm{S}}\right), h\left(\chi_{\mathrm{h}}\right)\right)$ and

$$
A\left(\chi_{\mathrm{S}}, \chi_{\mathrm{h}}\right)=\frac{6 \xi_{\mathrm{h}} \xi_{\mathrm{S}}}{M_{\mathrm{P}}^{2} \Omega^{4}} \frac{d S}{d \chi_{\mathrm{S}}} \frac{d h}{d \chi_{\mathrm{h}}} h S .
$$

In this work, we consider the Higgs field serves as inflaton for the $O(N)$ model and the $O(N \rightarrow N-1)$ model, which is ensured by $\xi_{h} \gg \xi_{S}$. We consider $\xi_{S}=0$ at the electroweak scale. In this situation, the kinetic terms of the scalar fields are canonical with $A\left(\chi_{\mathrm{S}}, \chi_{\mathrm{h}}\right)=0$, and the metric in this case is given by $\Omega^{2}=1+\left(\xi_{h} h^{2}+\xi_{S} S^{2}\right) / M_{\mathrm{pl}}^{2} \approx 1+\xi_{h} h^{2} / M_{\mathrm{pl}}^{2}$ with $S \sim 0$ [73,76-78].

The inflationary action in terms of the canonically normalized field $\chi$ is, therefore, given as

$$
S_{\mathrm{inf}}=\int d^{4} x \sqrt{\tilde{g}}\left[\frac{M_{\mathrm{p}}^{2}}{2} R+\frac{1}{2}(\partial \chi)^{2}-U(\chi)\right],
$$

with the potential in terms of the canonically normalized field $\chi$ as

$$
U(\chi)=\frac{\lambda_{h}(h(\chi))^{4}}{4 \Omega^{4}}
$$

where the new field $\chi$ are defined by

$$
\frac{d \chi}{d h} \approx\left(\left(1+\xi_{h} h^{2} / M_{\mathrm{p}}^{2}+6 \xi_{h}^{2} h^{2} / M_{\mathrm{p}}^{2}\right) /\left(1+\xi_{h} h^{2} / M_{\mathrm{p}}^{2}\right)^{2}\right)^{1 / 2}
$$

for $h-$ inflations [73]. Note that $\lambda_{h}$ and $\xi_{h}$ have a scale $(h)$ dependence. The potential of $U(\chi)$ at the high scale of $\chi \gg M_{P}$ should be flat enough to drive the slow-roll inflation.

The slow-roll parameters used to characterize the inflation dynamics are,

$\varepsilon(\chi)=\frac{M_{\mathrm{p}}^{2}}{2}\left(\frac{d U / d \chi}{U(\chi)}\right)^{2}, \quad \eta(\chi)=M_{\mathrm{p}}^{2}\left(\frac{d^{2} U / d \chi^{2}}{U(\chi)}\right)$.

The field value at the end of inflation $\chi_{\text {end }}$ is obtained when $\varepsilon=1$, and the horizon exit value $\chi_{\text {in }}$ can be calculated by assuming an e-folding number between the two periods,

$$
N_{\mathrm{e}-\text { folds }}=\int_{\chi_{\mathrm{end}}}^{\chi_{\mathrm{in}}} d \chi \frac{1}{M_{\mathrm{p}} \sqrt{2 \varepsilon}} .
$$

Then, one can relate the inflationary observables of spectrum index $n_{s}$ and the tensor to scalar ratio of $r$ with the slow-roll parameters at the $\chi_{\text {in }}$,

$$
n_{s}=1+2 \eta-6 \varepsilon, \quad r=16 \varepsilon .
$$

The Planck results set $n_{s}=0.9677 \pm 0.0060$ at $1 \sigma$ level and $r<0.11$ at $95 \% \mathrm{CL}$ [4] for $N_{\mathrm{e}}=60$. Meanwhile, the nonminimal gravity couplings $\xi_{h}$ can be determined using the constraint coming from CMB observations [4], with the amplitude of scalar spectrum fluctuations $\Delta_{\mathcal{R}}^{2}$ being calculated as

$$
\Delta_{\mathcal{R}}^{2}=\frac{1}{24 \pi^{2} M_{\mathrm{p}}^{4}} \frac{U(\chi)}{\varepsilon}=2.2 \times 10^{-9} .
$$

With which, one can obtain the slow-roll inflation favored parameter regions of $\lambda_{s}$ and $\lambda_{h s}$ for the fixed $\lambda_{h}$ in the $O(N)$ scenario, since the two quartic coupling contribute to the inflation potential indirectly through the RGEs as shown in Appendix A. For the $O(N \rightarrow N-1)$ scenario, the $m_{h_{2}}, v_{s}$ and the mixing angle of $\theta$ determine the couplings of $\lambda_{h, h s, s}$ through Eq. (8). For the two scenarios, the slow-roll parameters $r$ are all of order $\sim \mathcal{O}\left(10^{-2}\right)$. Previous inflation studies of Refs. [73,76] shows that the successful implementation of slow-roll Higgs or singlet inflation usually occurs with relatively smaller quartic scalar couplings of order $\mathcal{O}(0.1-1)$.

At last, we comment on the thermal history of the Universe. One can estimate the reheating temperature when the decay of the inflaton starts competing with expansion $\mathrm{H} \sim \Gamma_{h}$ for Higgs inflation [81],

$$
\rho=3 H^{2} M_{p}^{2}=3 \Gamma_{h}^{2} M_{p}^{2} \equiv \frac{\pi^{2} g_{*}}{30} T_{R}^{4},
$$

here $g_{*} \approx 100$ is the number of relativistic degrees of freedom in the Universe during the reheating epoch. 

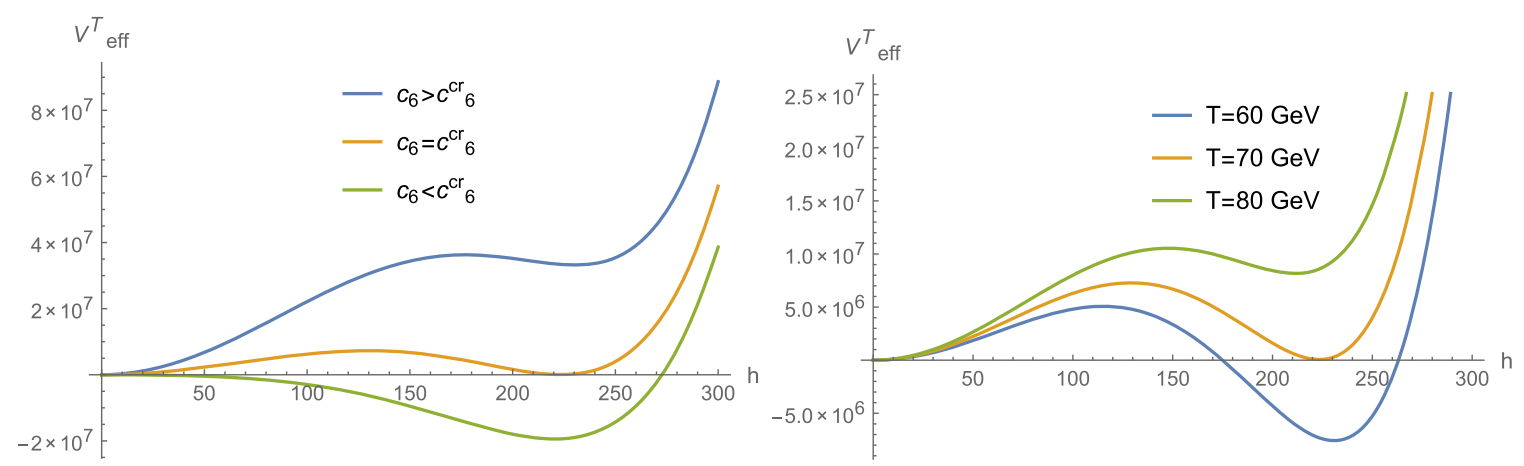

FIG. 6. The finite temperature effective potential v.s. dimension-six operator Wilson coefficients (temperature) with fixed temperature $T=70 \mathrm{GeV}$ (fixed $c_{6}=c_{6}^{c r}$ ) for left (right) panel.

The reheating temperature for the Higgs inflation was estimated through the parametric resonance of the oscillating Higgs field to W bosons (via $|H|^{2}|W|^{2}$ ) in Refs. [82,83]. Ref. [84] suggest $T_{R} \geq\left(\frac{15 \lambda_{h}}{8 \pi^{2} g_{*}}\right)^{1 / 4} \frac{M_{p}}{\xi_{h}}$. Within the inflation viable parameter spaces in $O(N \rightarrow N-1)$ scenarios under study, we have $\lambda_{h} \sim \mathcal{O}\left(10^{-4}-10^{-1}\right)$ and $\xi_{h} \sim$ $\mathcal{O}\left(10^{3}-10^{5}\right)$, therefore the $T_{R} \geq \mathcal{O}\left(10^{10}-10^{13}\right) \mathrm{GeV}$. For the $O(N)$ scenario, we have $\lambda_{h} \sim \mathcal{O}\left(10^{-2}-10^{-1}\right)$ and $\xi_{h} \sim \mathcal{O}\left(10^{5}\right)$, thus $T_{R} \geq \mathcal{O}\left(10^{13}\right) \mathrm{GeV}$. Freeze out of cold dark matter requires $x_{f} \equiv m_{\mathrm{DM}} / T_{f s} \approx 20$, and therefore the thermal history can occur as $T_{R}>T_{C}>T_{f s}>$ $T_{\mathrm{BBN}}$ to account for the EWPT and reheating as well as the successful Big Bang Nucleosynthesis (BBN) (with a typical temperature of a few $\mathrm{MeV}$ ). The freeze-out temperature $T_{f s}$ being smaller than the SFOEWPT temperature $T_{C}$, set the $m_{\mathrm{DM}}<20 T_{C} \sim 2 \mathrm{TeV}$ with $T_{C}$ being around $\sim \mathcal{O}\left(10^{2}\right) \mathrm{GeV}$.

\section{B. Electroweak phase transition}

With the temperature cooling down, the Universe can evolve from symmetric phase to the symmetry broken phase. The behavior can be studied with the finite temperature effective potential with particle physics models [85]. Through which one can obtain the critical classical field value and temperature being $v_{C}$ and $T_{C}$. Roughly speaking, a SFOEWPT can be obtained when $v_{C} / T_{C}>1$, then the electroweak sphaleron process is quenched inside the bubble and therefore one can obtain the net number of baryon over anti-baryon in the framework of EWBG. For the uncertainty of the value and possible gauge dependent issues we refer to Ref. [86]. The finite temperature effective potential includes the tree level scalar potential, the Coleman-Weinberg potential, and the finite temperature corrections [87]. For the finite temperature corrections, we adopt the method of Refs. [85,88] with the Espinosa approach [87]. Then the critical parameters of EWPT can be calculated when there are two degenerate vacuums with a potential barrier. Due to rich vacuum structures of the potential at finite temperatures, there can be one-step or multi-step phase transitions. A SFOEWPT can occur at the first or the second step in the two-step scenario. We will investigate one-step and two-step phase transitions in the $O(N)$ and the $O(N \rightarrow N-1)$ scenarios.

Before the detailed study, we first warm up by briefly recalling the one-step SFOEWPT condition on Wilson coefficients of the dimension-six operator $[59,89,90]$,

$$
\frac{m_{h}^{2}}{3 v^{4}}<\frac{c_{6}}{\Lambda^{2}}<\frac{m_{h}^{2}}{v^{4}} .
$$

The left panel of Fig. 6 shows the potential shape at critical temperature with different dimension- six operator Wilson coefficients and depicts that a suitable $c_{6}=c_{6}^{c r}$ is needed to obtain a proper vacuum barrier to separate two degenerate vacua at critical temperature $T_{c}$, therefore making the SFOEWPT feasible. On the right panel of Fig. 6, we plot the finite temperature effective potential as a function of temperature for fixed $c_{6}$, one can find that the symmetry will be restored at high temperature and break at temperature lower than the critical temperature $T_{c}$. It should be noted that, with the spontaneous symmetry breaking of $O(N \rightarrow N-1)$, the two contributions of the $c_{6}$ from $s h^{2}$ and $s^{3}$ terms cancel out each other [61], and therefore the tree-level induced dimension-six operator disappears, which is the same as in the $\mathrm{SM}+1$ singlet case being studied in Ref. [66]. In this case, the dimension-six operator shows up at loop level which is too small to affect the EWPT dynamics. This property can explain why the $\mathrm{SM}+1$ singlet scalar with $Z_{2}$ does not prefer one-step SFOEWPT, and here one may need to pursue the two-step types where the DM can be useful for achieving a SFOEWPT $[46,91-93]$. In this work, we reconfirm the same property in the $O(N)$ and $O(N \rightarrow N-1)$ cases.

Following the approach of Ref. [88], the finite temperature of $V(h, S(s), N, T)=V_{0}(h, S(s))+V_{C W}(h, S(s), N)+$ $V_{T}(h, S(s), N, T)+V_{\text {daisy }}(h, S(s), N, T)$ for $O(N)(O(N \rightarrow$ $N-1)$ ) case is adopted to estimate the order parameters of the SFOEWPT, with $V_{0}, V_{C W}, V_{T}$, and $V_{\text {daisy }}$ being zero-temperature tree-level potential, one-loop ColemanWeinberg potential, finite-temperature potential, and daisy 


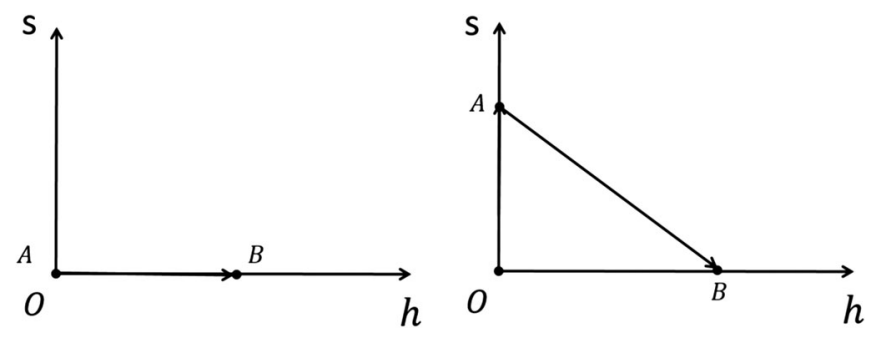

FIG. 7. One- and two-step EWPT types in $\mathrm{O}(\mathrm{N})$ for left and right panels, respectively.

terms. These functions for the $O(N)(O(N \rightarrow N-1))$ case are given in Appendix B. In the case of $O(N)$ scalars, the corresponding critical temperature and critical field value for one- and two-step EWPT types (see Fig. 7) can be evaluated through the following degeneracy conditions,

$$
\begin{aligned}
V\left(0,0, N, T_{C}\right) & =V\left(h_{C}^{B}, 0, N, T_{C}\right), \\
\left.\frac{d V\left(h, 0, N, T_{C}\right)}{d h}\right|_{h=h_{C}^{B}} & =0,
\end{aligned}
$$

and

$$
\begin{aligned}
V\left(0, s_{C}^{A}, N, T_{C}\right) & =V\left(h_{C}^{B}, 0, N, T_{C}\right), \\
\left.\frac{d V\left(h, 0, N, T_{C}\right)}{d h}\right|_{h=h_{C}^{B}} & =0 .
\end{aligned}
$$

Here the $s_{C}$ is the $O(N)$ broken direction, which is analogous to the $O(N \rightarrow N-1)$ scenario. The survey of the one-step EWPT in the $O(N)$ scenario shows that the quartic coupling between the SM Higgs and the $O(N)$ scalars $S_{i}\left(\lambda_{h s}\right)$ should be large enough in order to make the SFOEWPT occurs, which is not favored by the slow-roll Higgs inflation. Generally, within the parameter spaces of a large $\lambda_{h s}$ where one can have a SFOEWPT and the inflation is invalid, the perturbativity of scalar quartic coupling and unitarity are violated due to the $\mathrm{RG}$ running of couplings as explored in Sec. IVA.

We demonstrate the one-step and two-step phase transition patterns in Fig. 8. The one-step EWPT types in $O(N \rightarrow N-1)$, occurs along the $\overrightarrow{O B}$ line, and the two-step EWPT occurs through the process of $O \rightarrow A \rightarrow B$. With
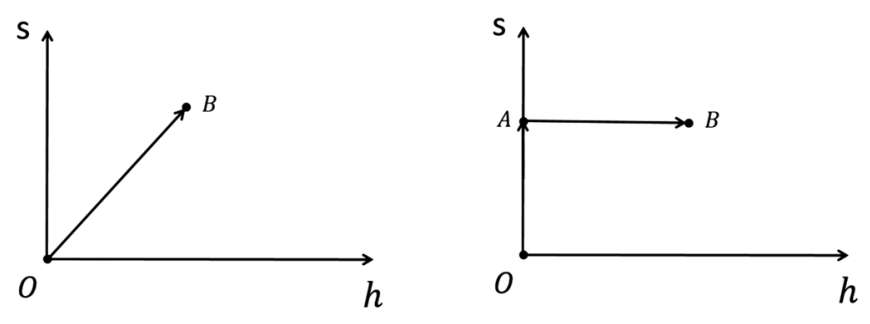

FIG. 8. One- and two-step phase transition types in $O(N \rightarrow$ $N-1)$ scenario for left and right panels. two degenerate vacuums being separated by a potential barrier structures at the critical temperature, the degeneracy conditions can be expressed as Eqs. (49) and (50).

$$
\begin{aligned}
V\left(0,0, N, T_{C}\right) & =V\left(v_{C}^{B}, s_{C}^{B}, N, T_{C}\right), \\
\left.\frac{d V\left(h, s, N, T_{C}\right)}{d h}\right|_{h=h_{C}^{B}, s=s_{C}^{B}} & =0, \\
\left.\frac{d V\left(h, s, N, T_{C}\right)}{d s}\right|_{h=h_{C}^{B}, s=s_{C}^{B}} & =0 . \\
V\left(0, s_{C}^{A}, N, T_{C}\right) & =V\left(h_{C}^{B}, s_{C}^{B}, N, T_{C}\right), \\
\left.\frac{d V\left(h, s, N, T_{C}\right)}{d h}\right|_{h=h_{C}^{B}, s=s_{C}^{B}} & =0, \\
\left.\frac{d V\left(h, s, N, T_{C}\right)}{d s}\right|_{h=h_{C}^{B}, s=s_{C}^{B}} & =0 .
\end{aligned}
$$

\section{Dark matter/radiation}

When the $O(N)$ is kept at zero temperature, the $N$-singlet scalars can all serve as dark matter candidates. Suppose gravity violates global symmetries, then the Goldstone boson may acquire a mass through nonpertubative gravitational effects $[94,95]$. The nonperturbative gravity effects can break the $O(N)$ symmetry at $M_{P}$ scale through the lowest high-dimension operators, i.e., dimension-five operators, induce mass terms to Goldstone bosons and make $N-1$ majoron like particles,

$$
\frac{C_{1}\left(H^{\dagger} H\right)^{2} s_{i}}{M_{P}}+\frac{C_{2}\left(H^{\dagger} H\right) s_{i}^{3}}{M_{P}}+\frac{C_{3} s_{i}^{5}}{M_{P}} .
$$

For the wilson coefficients $C_{i} \sim \mathcal{O}(1)$ and the VEV of scalar singlet $v_{s} \sim \mathcal{O}\left(10^{3}\right) \mathrm{GeV}$, one can expect the masses of majoron like particle,

$m_{s_{1, \ldots, N-1}}=\frac{16 C_{1} v^{4}+12 C_{2} v^{2} v_{s}^{2}+5 C_{3} v_{s}^{4}}{2 M_{P} v_{s}} \sim \mathcal{O}(1) \mathrm{eV}$.

In this mass region, we can expect the majoron decaying to diphoton through the nonminimal gravity couple term which breaks the $O(N-1)$ symmetry as in the Ref. [96]. We found the Goldstone bosons here is long-lived, with $\tau \sim \Gamma^{-1} \sim 10^{46} s$, they can survive until the recombination era and may contribute to the Universe radiation density at the time of recombination or $\mathrm{BBN}$.

\section{NUMERICAL RESULTS}

\section{A. $O(N)$ scenario}

We first explore inflation dynamics without taking into account the Higgs precision bounds. In Fig. 9, we show the 

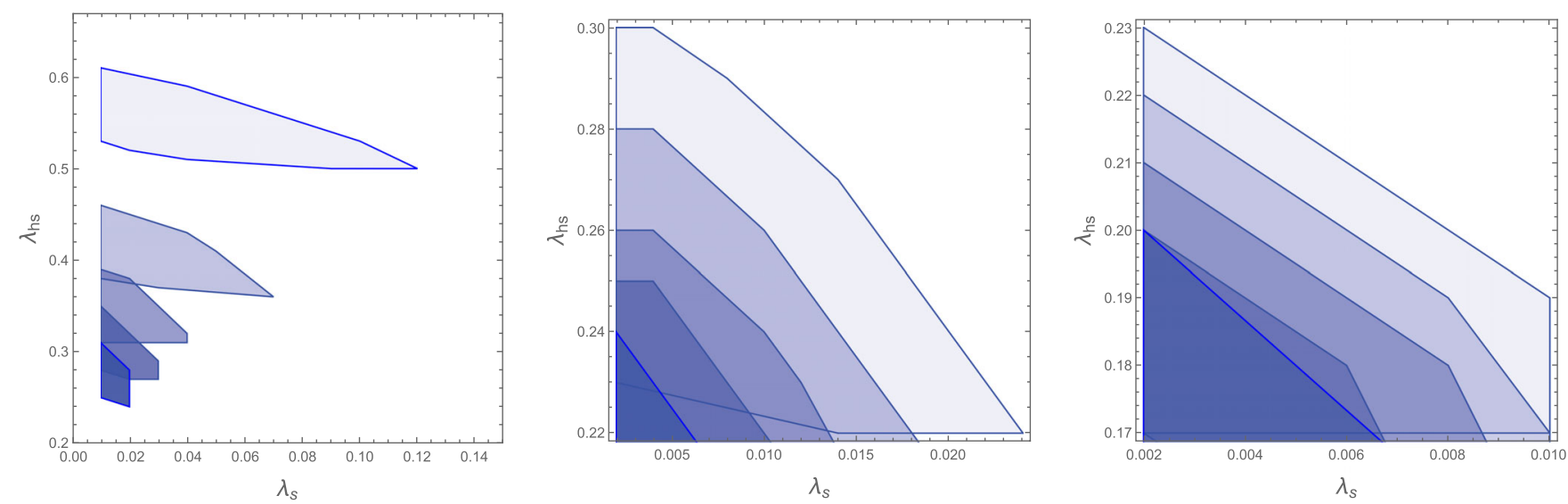

FIG. 9. Inflation feasible parameters plane of $\left(\lambda_{s}, \lambda_{h s}\right)$ for different $\mathrm{N}$ within $O(N)$ scalar model, a larger $N$ is shown by a deeper color, and the corresponding $N$ are $1 \rightarrow 5,6 \rightarrow 10$ and $11 \rightarrow 15$ for left, middle and right panels, respectively. Note that the shaded regions are allowed rather than excluded.
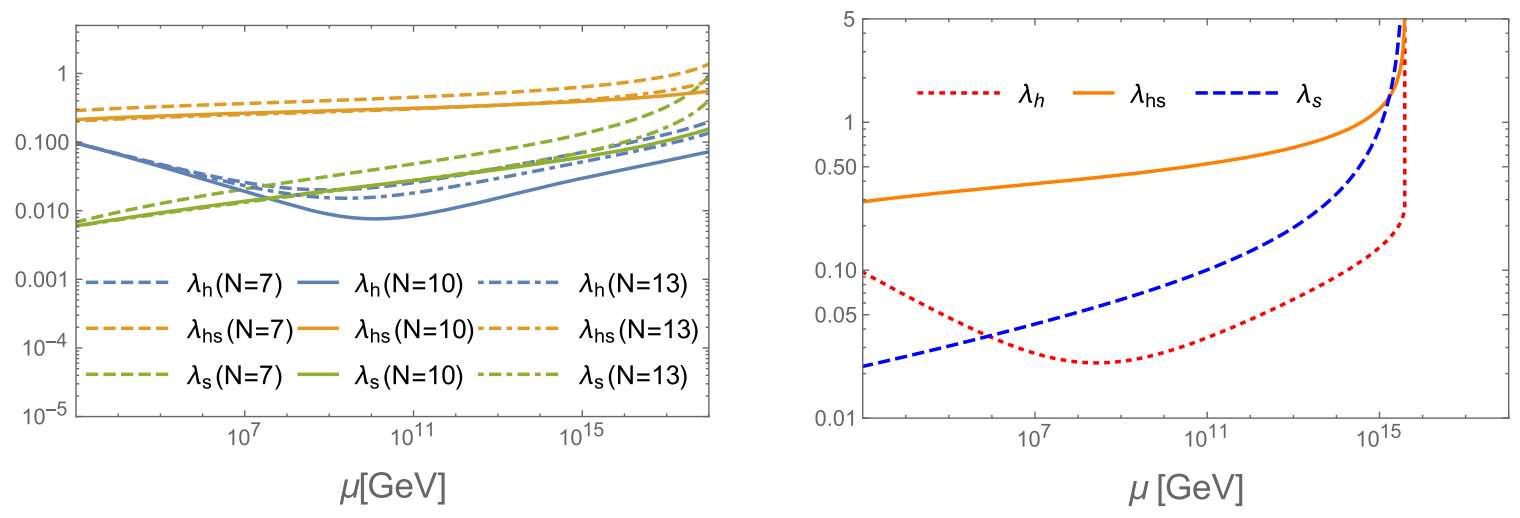

FIG. 10. RG running of $\lambda_{s}, \lambda_{h}$ and $\lambda_{h s}$. Left: the scalar quartic couplings where the inflation is valid. Right: the scalar quartic couplings lives in the parameter region where the inflation is invalid.

Higgs inflation feasible parameter regions in the plane of $\left(\lambda_{s}, \lambda_{h s}\right)$ after imposing the theoretical constraints up to Planck scale as aforementioned in Sec. II A. Where, the upper and lower bounds of $\lambda_{h s}$ are mostly coming from

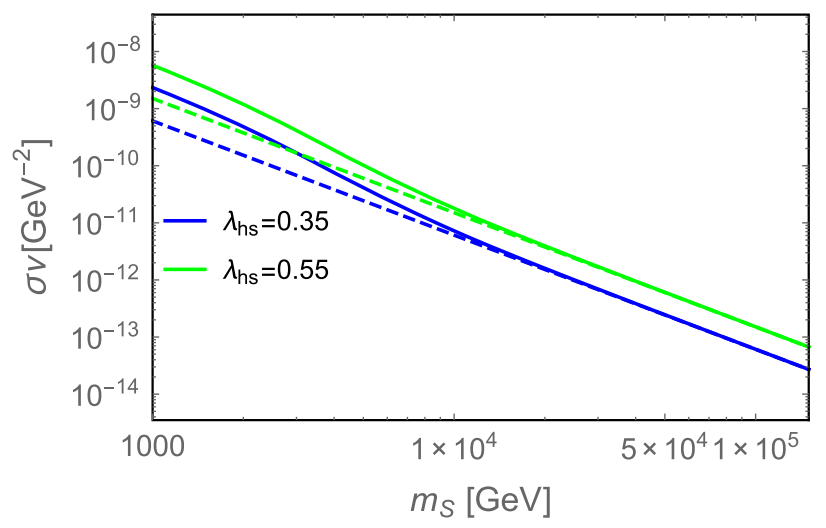

FIG. 11. DM annihilation cross section with the dashed lines indicating the seagull diagram contribution and the solid lines being the all annihilation channels contributions. perturbativity and unitarity, and stability conditions. The inflation feasible range in the plane of $\left(\lambda_{s}, \lambda_{h s}\right)$ is largest when $N=1$. The feasible ranges diminish with the increase of $N$ and are overlapped for the two neighbor $N$ expect for $N=1$ and $N=2$. The decrease of the inflation valid area with the increase of $N$ is due to the fact that: a larger $\mathrm{N}$ leads to more contributions of $\lambda_{h s}$ to $\lambda_{h}$ at the inflation scale through RG running (using the RGEs given in Appendix A), and therefore the stability, perturbativity and unitarity set the lower and upper bounds of $\lambda_{h s}$. We plot the RG running of the scalar quartic couplings for the case of $N=7,10,13$ in Fig. 10. The perturbativity of quartic couplings and the unitarity can be violated due to RG running of couplings as shown in the right panel of Fig. 10.

With thermal averaged annihilation cross sections being the same as in Ref. [73] for each $S_{i}$, see Appendix C, and using Lee-Weinberg method [97], we can estimate $\Omega^{S_{i}} h^{2} \sim$ $1 / \sigma v_{\text {rel }} \sim m_{S_{i}}^{2} / \lambda_{h s}^{2}$ for a large dark matter mass. Previous studies show that the mass region of Higgs-portal real 1-singlet scalar DM case is excluded up to $\sim \mathrm{TeV}$ scale by Xenon1T [45,98]. 

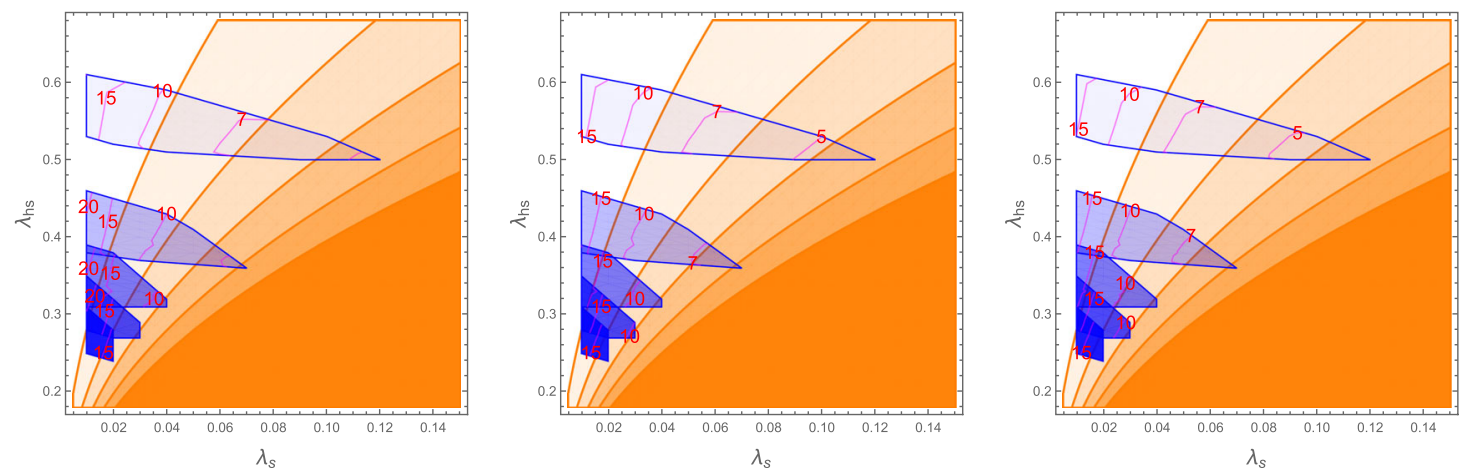

FIG. 12. Colliders, EWPOs, and inflation constraints on $m_{S_{i}}$ in the $\left(\lambda_{s}, \lambda_{h s}\right)$ plane for different $N$. The magenta line is the allowed magnitude of $m_{S_{i}}$ [TeV] by the CEPC, ILC, and FCC-ee from left to right panel. Blue regions is for the feasible inflation within $O(N)$ model, orange regions represent the allowed regions by EWPOs confine. For both two color-codes, a deeper color corresponds to a larger $N$ with $N=1,2,3,4,5$.

It should be noted that the future Linear collider constraints would limit the dark matter mass to be $\mathrm{TeV}$ scale $(\sim O(1-10) \mathrm{TeV})$ in the inflation feasible region as shown in Fig. 12. In this case, the seagull diagram dominates the contributions to the dark matter pair annihilations. We show the annihilation cross section in Fig. 11 to illustrate that. It's easy to see that the contributions of the Eq. (C1) or Eq. (53) would oversaturate the relic abundance for the inflation feasible $\lambda_{h s}$ though the highly suppress of $\sigma^{S I}$ by large $m_{S}$ make the mass region safe from Xenon 1T.

For $m_{\mathrm{DM}}>10 \mathrm{TeV}$, one obtains $T_{f s} \sim m_{\mathrm{DM}} / x_{f}>$ $500 \mathrm{GeV}$, therefore the dark matter freeze out happens earlier than the EWPT, and thus only the seagull diagram process can happen with the Higgs finite states have effectively zero masses. Then, the annihilation cross section of Eq. (C1) reduces to

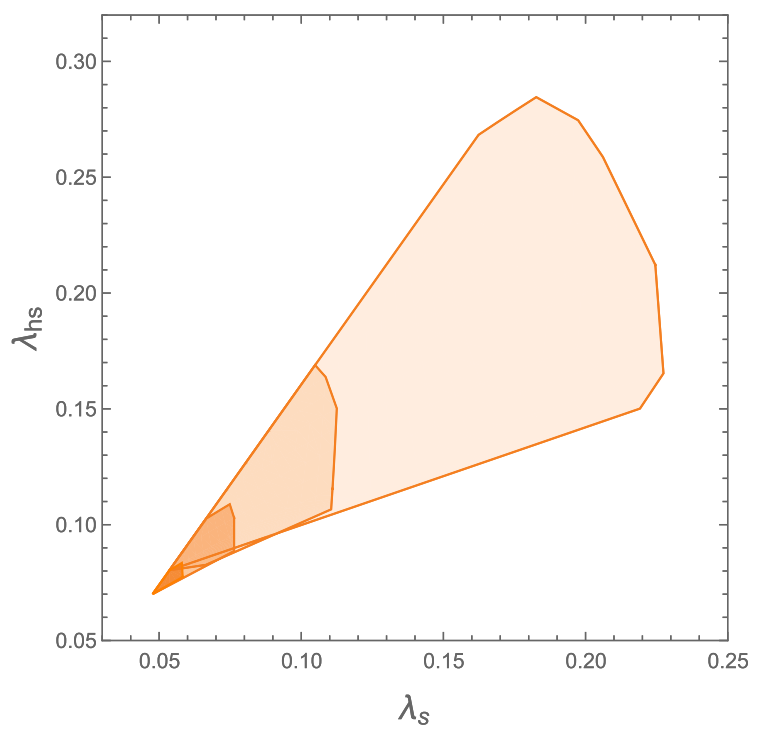

$$
\left\langle\sigma v_{\text {rel }}\right\rangle_{h h}=\frac{\lambda_{h s}^{2}}{64 \pi m_{s}^{2}}
$$

Here we point out that $m_{s} \sim \mu_{s}$ due to the electroweak symmetry is still kept at temperature higher than $T_{c}$ within the framework of EWBG. Then, if the relic abundance is partially saturated by $S_{i}$, one needs a larger H-S quartic coupling $\lambda_{h s}$. The large $m_{S}$, in fact, would decouple from the phase transition has been studied in Ref. [50]. Furthermore, the largeness of the $\lambda_{h s}$ may result in the perturbativity and unitarity problem of quartic couplings $\lambda_{h, h s}$, as shown in Fig. 10 (after taking into account the RG running effects), and therefore shut down the possibility to explain inflation.

We briefly summarize this section as follows. The numerical survey of the two step EWPT shows that a SFOEWPT requires $\mathrm{N} \geq 7$ in the inflation favored

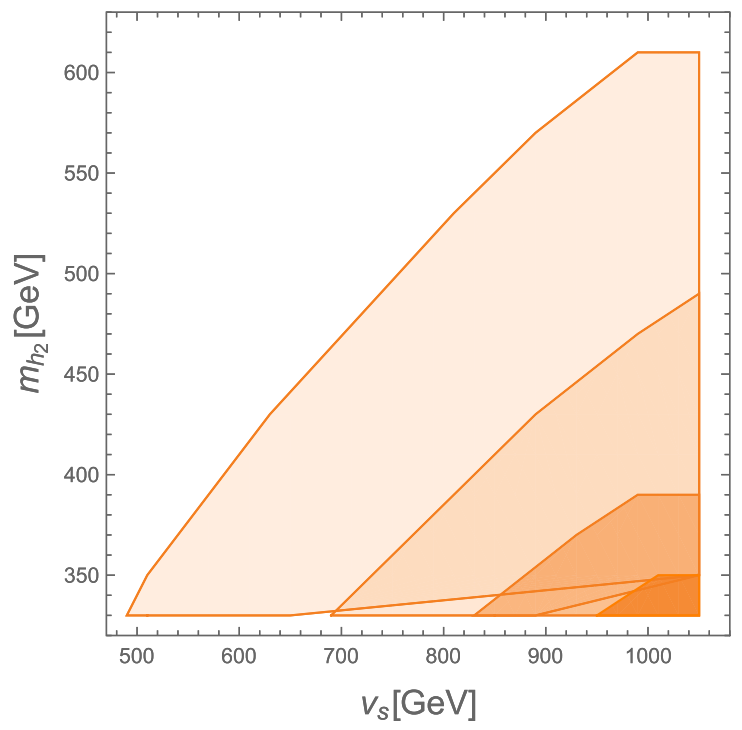

FIG. 13. Inflation feasible parameter planes of $\left(\lambda_{s}, \lambda_{h s}\right)$ and $\left(v_{s}, m_{h_{2}}\right)$ for different $N$ within $O(N \rightarrow N-1)$ scalar model, a deeper color corresponds to a larger $N$, the corresponding $N$ are $1,2,3$, and 4 , respectively. 


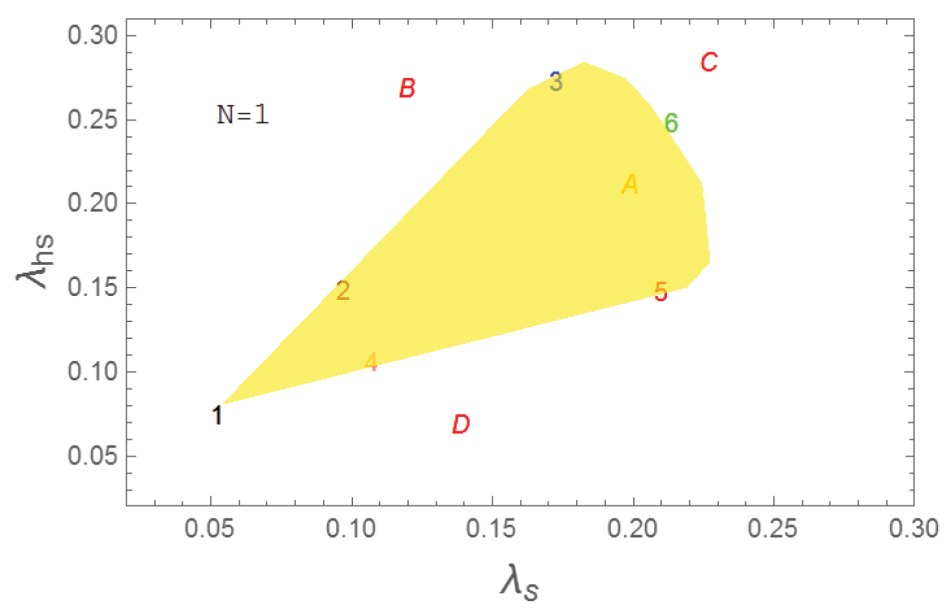

$1 m_{h_{2}}=330[\mathrm{GeV}], v_{s}=1000[\mathrm{GeV}]$

$2 m_{h_{2}}=450[\mathrm{GeV}], v_{s}=1000[\mathrm{GeV}]$

$3 m_{h_{2}}=600[\mathrm{GeV}], v_{s}=1000[\mathrm{GeV}]$

$4 \quad m_{h_{2}}=330[\mathrm{GeV}], v_{s}=700[\mathrm{GeV}]$

$5 m_{h_{2}}=330[\mathrm{GeV}], v_{s}=500[\mathrm{GeV}]$

$6 m_{h_{2}}=500[\mathrm{GeV}], v_{s}=750[\mathrm{GeV}]$

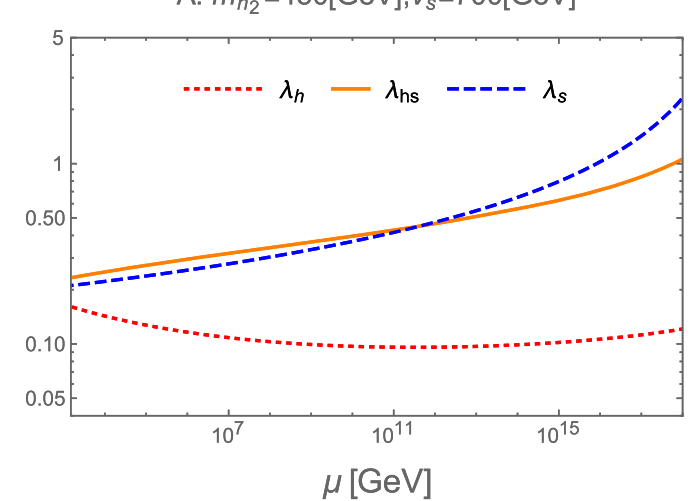

C: $m_{h_{2}}=550[\mathrm{GeV}], v_{s}=800[\mathrm{GeV}]$

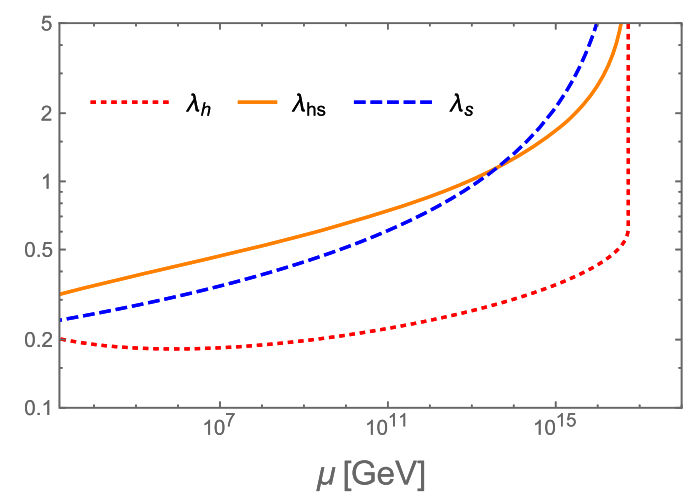

B: $m_{h_{2}}=700[\mathrm{GeV}], v_{s}=1400[\mathrm{GeV}]$

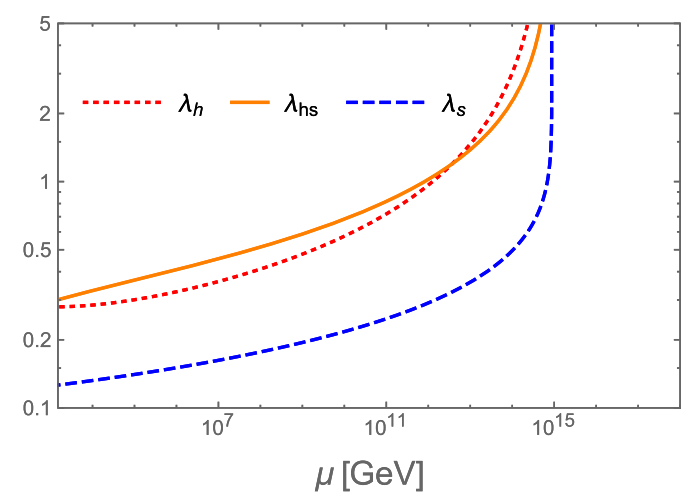

D: $m_{h_{2}}=230[\mathrm{GeV}], v_{s}=430[\mathrm{GeV}]$

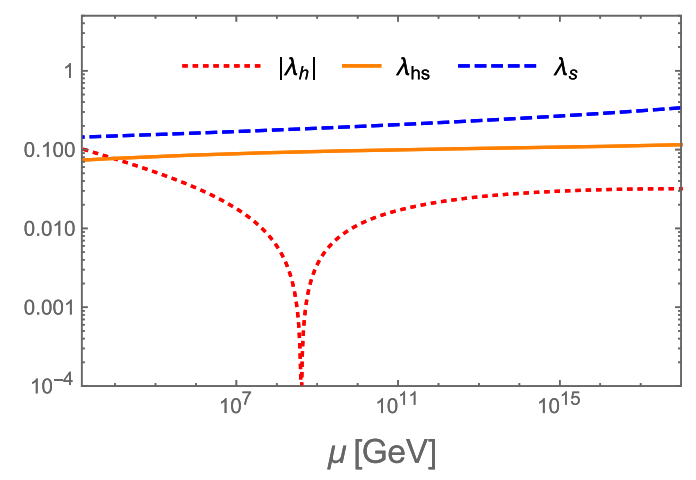

FIG. 14. Top panel: the Higgs inflation feasible parameter spaces of $\left(\lambda_{s}, \lambda_{h s}\right)$. Middle and bottom panels: four samples of the RG running coupling $\left(\lambda_{h, h s, s}\right)$.

parameter regions. Unfortunately, as can be seen from Fig. 12, the inflation valid $N$ is bounded to be $N<4$ after imposing the constraints from EWPOs, which therefore shuts down the window to realize the SFOEWPT. The $c_{H}^{N} v^{2} / m_{S_{i}}^{2}$ [the $c_{H}^{N}$ is given by Eq. (13)] is bounded to be smaller than $0.0038,0.0034$, and 0.0028 by CEPC with the luminosity of $5 \mathrm{ab}^{-1}$, ILC with all center-of-mass energies, and FCC-ee with the luminosity of $10 \mathrm{ab}^{-1}$, with which we can obtain the bounds on the hidden scalar masses of $m_{S_{i}} \sim \mathcal{O}(1-10) \mathrm{TeV}$. In this mass region, the $N$ scalars cannot explain the correct DM relic density.

\section{B. $O(N \rightarrow N-1)$ scenario}

As in the previous section, here we first perform the inflation analysis before considering the Higgs precision. We show the Higgs inflation valid parameter spaces in Fig. 13, and the feasible ranges diminish with the increase of $N$ and are overlapped for the two contiguous $N$. The left panel indicates that sthe magnitude of $\lambda_{h s}$ increases with the increase of $\lambda_{s}$, which is different from the $O(N)$ scalar model scenario being explored in the previous section. An interesting triangular shape shows up due to the bounds on $m_{h_{2}}$ and $v_{s}$ from perturbativity, unitarity, and stability, 

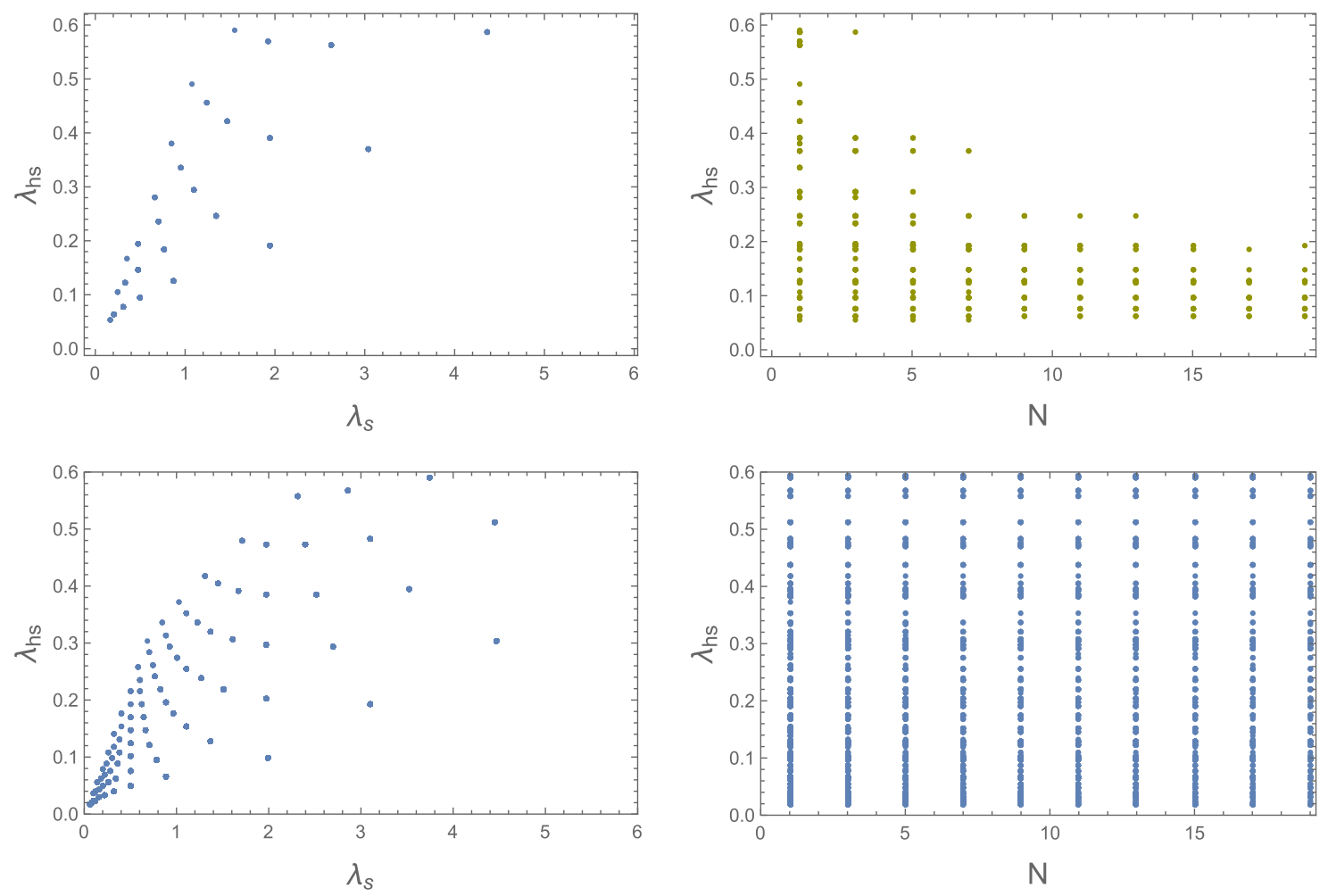

FIG. 15. One-step (top panel) and two-step (bottom panel) SFOEWPT valid points within the $O(N \rightarrow N-1)$ scenario.

together with the relations among quartic couplings, the Higgses masses and VEVs. Here, it should be noted that the lower bound of $m_{h_{2}}$ is set by the stability bounds. The scalar quartic Higgs coupling will be negative for $m_{h_{2}}<330 \mathrm{GeV}$, this set the lower boundary of the inflation valid parameter region. The upper bound is set by the magnitude of the $v_{s}$ assisted by the $m_{h_{2}}$, and the upper bound on $m_{h_{2}}<600 \mathrm{GeV}$ is to fulfill the perturbativity and unitarity conditions at high scale. With the increase of $\mathrm{N}$, the perturbativity and unitarity conditions, together with stability requirement results in a

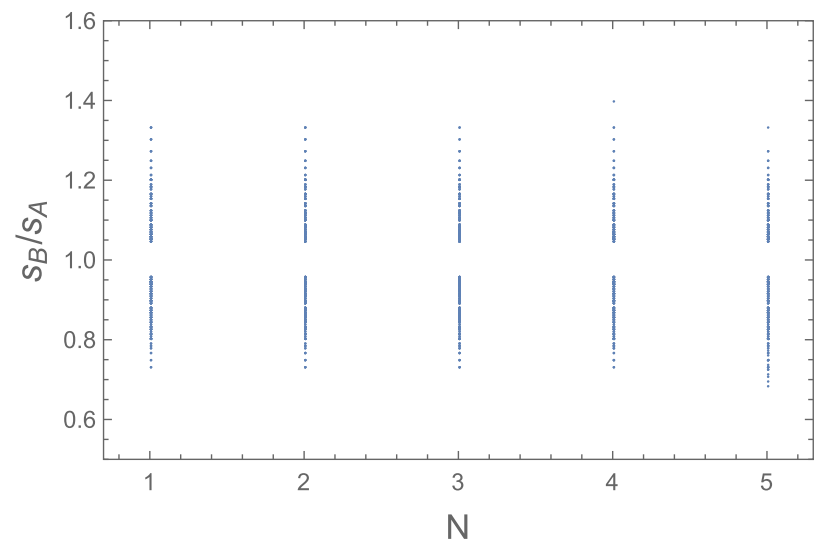

FIG. 16. The two-step SFOEWPT points in $O(N \rightarrow N-1)$ model. smaller parameter region of $\lambda_{h s}$ and $\lambda_{s}$ as shown in Fig. 13. The slow-roll inflation is almost excluded for $N \geq 5$.

To explain the property more transparently, we plot it in Fig. 14 by taking the $N=1$ case as an example. In the upper plot, the yellow region stands for the inflation feasible region for $N=1$ in the $\left(\lambda_{s}, \lambda_{h s}\right)$ plane within the $O(N \rightarrow N-1)$ scalar model with the number of Goldstones being zero, the values of $m_{h_{2}}$ and $v_{s}$ for the numbers and alphabets can be found at the right hand of the upper, middle and bottom panels, respectively. The middle and bottom panels show the RG running of couplings (for the points A, B, C, and D) from electroweak scale to Planck scale. Note that, we use an absolute value for the coupling $\lambda_{h}$ in the last figure, the downward tip there means the stability is violated at the point of $\mathrm{D}$. That indicates that the stability is the lower bound for $\left(\lambda_{s}, \lambda_{h s}\right)$ plane in the $O(N \rightarrow$ $N-1)$ scenario. For B and C points, the perturbativity and unitarity are violated due to the RG running of couplings. This indicates that the perturbativity and the unitarity set the upper bound.

Now, we explore the EWPT property for the $O(N \rightarrow$ $N-1)$ scenario. For the one-step case, one can realize a SFOEWPT with a small $\lambda_{h s}$ with increasing $N$, as shown in Fig. 15, which means that the Goldstone contribution to the EWPT is notable, although this property disappears in the two-step scenario as can be seen in the bottom-right panel. The results show that different from the one-step situation, the SFOEWPT occurs more easily with a relatively small 

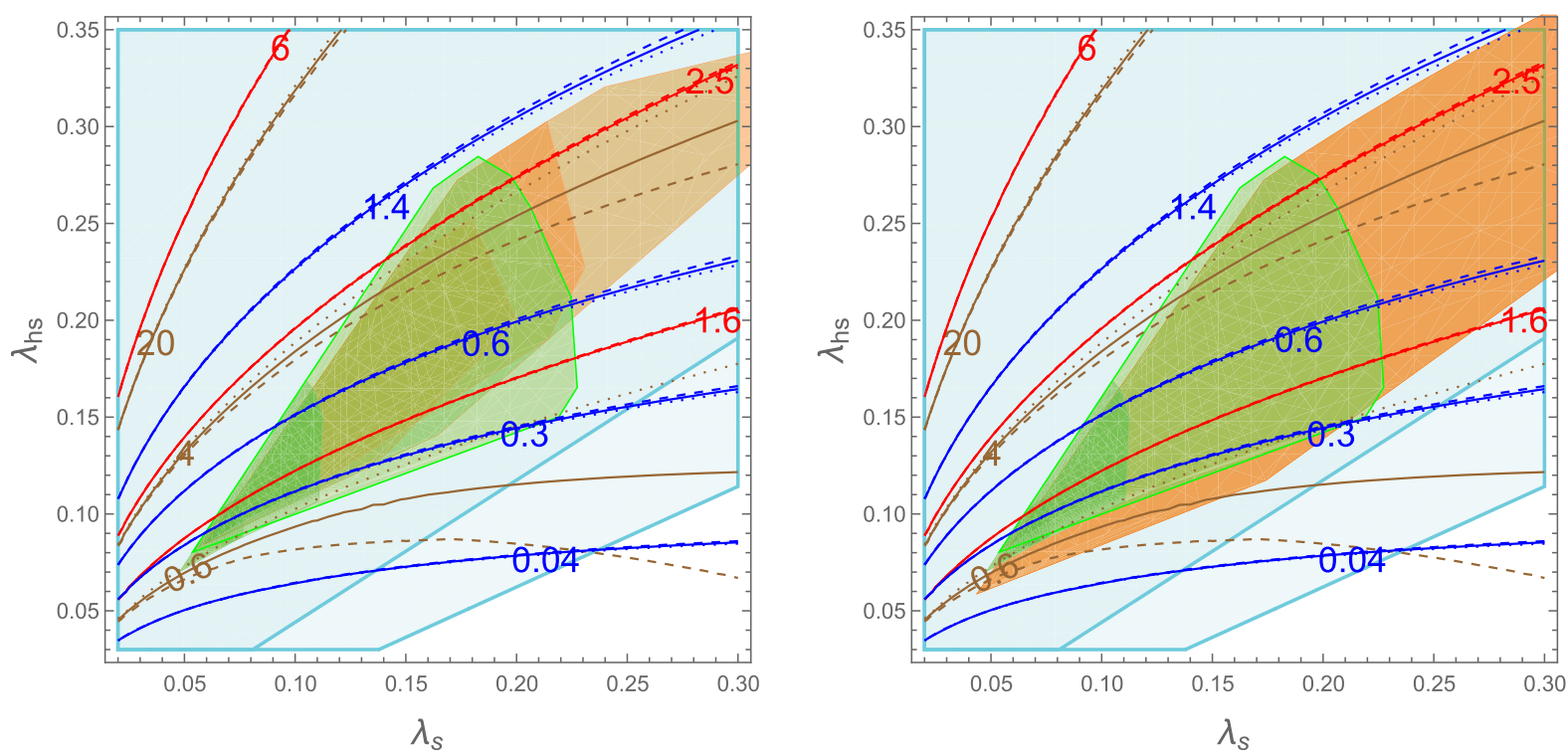

FIG. 17. The $O(N \rightarrow N-1)$ scenario. The $\lambda_{h_{1} h_{1} h_{1}}, \lambda_{h_{2} h_{1} h_{1}}$ (both normalized by $\left.\lambda_{h h h}^{\mathrm{SM}}\right)$, and $\Gamma_{h_{2}}^{\mathrm{tot}}$ are shown by red, blue, and brown contours, respectively. Dashed, solid, and dotted lines stand for $N=1,2,3$, respectively. Cyan regions present the allowed regions by the invisible Higgs decay bounds from $B_{\text {inv }}<0.34$ [67], in which the light and deep colors correspond to $N=2,3$, respectively. The feasible regions of inflation are shown by green color regions, and the orange regions represent one- and two-step SFOEWPT for the left and right panels, respectively. For those colors, a deeper color corresponds to a larger $N$ for $N=1,2,3$.

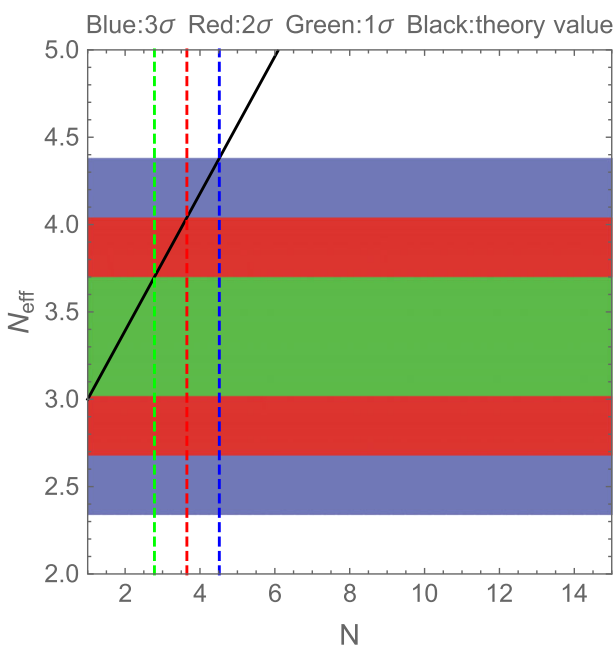

FIG. 18. The Goldstone faked effective neutrino number.

value of the $\lambda_{h s}$ for the two-step case. Moreover, our study demonstrates that the rate of $s_{B} / s_{A}$ can be larger or smaller than 1 for different $N$, as shown in Fig. 16, which reconfirms the study of Ref. [45].

In Fig. 17, we show the parameter regions that can accommodate successful Higgs inflation and a SFOEWPT together. The Higgs cubic couplings and Higgs decay widths in the $O(N \rightarrow N-1)$ model are also shown. The inflation and one(two)-step SFOEWPT are allowed by the Higgs invisible decay bounds from LHC [67] for different $N$, which is marked by cyan. As shown in Fig. 16, the twostep SFOEWT shows no obvious relation with $N$ due to $s_{A}$ can be higher or lower than $s_{B}$. For $N>1$, the slow-roll Higgs inflation does not occur in some SFOEWPT allowed parameter spaces with relatively large quartic couplings, this property is caused by the bound on $m_{h_{2}}$ and $v_{s}$ from perturbative unitarity and stability (from electroweak scale to Planck scale). For $1<N \leq 3$, more parameter spaces of $\left(\lambda_{s}, \lambda_{h s}\right)$ are allowed by two-step SFOEWPT condition in comparison with the one-step SFOEWPT condition. The ratio of the triple Higgs couplings $\left(r_{3 h_{1}}=\lambda_{h_{1} h_{1} h_{1}} / \lambda_{h h h}^{\mathrm{SM}}\right.$ and $r_{h_{2} h_{1} h_{1}}=\lambda_{h_{2} h_{1} h_{1}} / \lambda_{h h h}^{\mathrm{SM}}$ ) increase with the increase (decrease) of $\lambda_{h s}\left(\lambda_{s}\right)$. With the SM Higgs resonance search using the SM Higgs pairs production process, one can estimate the cross section with respect to the SM case being $\sigma_{h_{1} h_{1}} / \sigma_{h h}^{\mathrm{SM}} \sim \cos ^{2} \theta \times r_{3 h_{1}}^{2} \times \Gamma_{h_{1}}^{\mathrm{tot}} / \Gamma_{h}^{\mathrm{SM}} \sim 0.98^{2} \times 2^{2} \sim 3.8$ and therefore a large enhancement of the cross section can be expected. With increasing of $N$, one can expect the ratio $\sigma_{h_{1} h_{1}} / \sigma_{h_{1} h_{1}}^{\mathrm{SM}}$ decreasing due to the decrease of $r_{3 h_{1}}$. With increasing of $\lambda_{h s}$, the triple Higgs coupling of $\lambda_{h_{2} h_{1} h_{1}}$ varies in the range of $[0.3,1.4]$ in units of $\lambda_{3 h}^{\mathrm{SM}}$. The cross section of the heavy Higgs is $\sigma_{h_{1} h_{1}}^{h_{2}} \sim\left(\sqrt{2} m_{t} / v\right)^{2} \sin ^{2} \theta \times \lambda_{h_{2} h_{1} h_{1}}^{2} /$ $\left(m_{h_{2}} \Gamma_{h_{2}}^{\text {tot }}\right)$. Due to the $\Gamma_{h_{2}}^{\text {tot }} \ll m_{h_{2}}$, the resonance interference explored in Ref. [66] can be safely neglected here. We postpone the detailed collider search of the parameter spaces to a separate publication.

This part of the study constitutes one of our main results: building a connection between the inflation/ EWPT and the Goldstone numbers $(N-1)$ of the spontaneously broken global symmetry group. The feature being studied here can exist in other hidden sectors of extended 


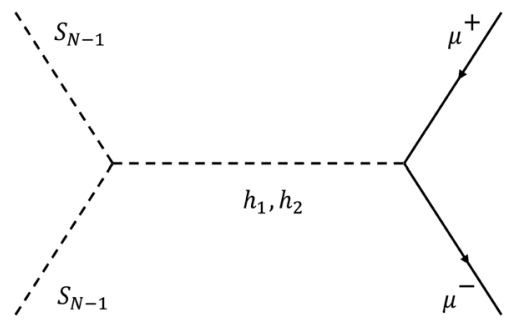

FIG. 19. Goldstone annihilation process.

SM, provided the hidden sectors respect a global symmetry that will be broken into subsets, wherein the remnant Goldstones will contribute to the thermal effective potential and the RGEs of scalar quartic couplings (for the inflationary potential).

Since the Goldstone might fake the effective neutrino and contribute to the dark radiation, the number of Goldstones will be limited by related experiments. The effective neutrino pling temperature as $[99,100]$,

$$
N_{\mathrm{eff}}=3\left(1+\frac{\Delta N_{S_{N-1}}}{3}\left(\frac{g_{*}\left(T_{\nu}^{d}\right)}{g_{*}\left(T_{S_{N-1}}^{d}\right)}\right)^{4 / 3}\right)
$$

with $\Delta N_{s_{N-1}}=4(N-1) / 7$ due to there is $(N-1)$ Goldstone bosons decoupled at $T>T_{S_{N-1}}^{d}$ and present before the recombination eras, the effective number of relativistic degrees of freedoms are $g_{*}\left(T_{\nu}^{d}\right)=43 / 4$ and $g_{*}\left(T_{s_{N-1}}^{d}\right)=$ $57 / 4$ supposing that the Goldstone bosons decouple just before muon annihilation. One can constrain the number of Goldstones as in Fig. 18 using the recent $1 \sigma$ experimental at $3 \sigma$.

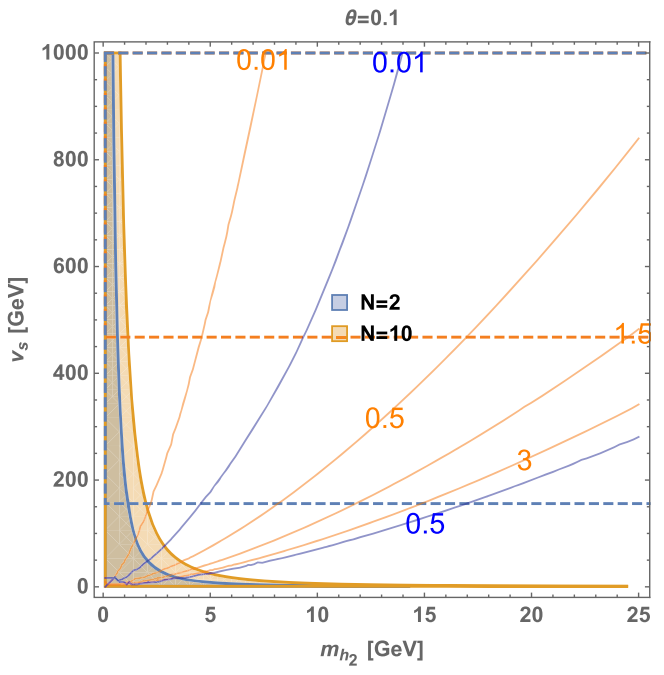
number can be expressed in terms of the Goldstone decoudata $N_{\text {eff }}=3.36 \pm 0.34$ [101], which depicts that $N \leq 4$

We study how the Goldstone decouples from the thermal bath and consider the possibility of the Goldstones contributing to the dark radiation following Ref. [102]. For the heavy Higgs contributions are typically small, one needs to focus on the light Higgs case alternatively, cf., $m_{h_{2}}<2 m_{h_{1}}$. When the decay width $\Gamma_{h_{2}} \ll m_{h_{2}}$ in the small mass region of $m_{h_{2}}$, the cross section of the Goldstone annihilating to $\mu^{+} \mu^{-}$(as shown in Fig. 19) is given by

$\langle\sigma v\rangle_{s_{N-1} s_{N-1} \rightarrow \mu^{+} \mu^{-}}=\frac{\lambda_{h s}^{2}}{128 \pi} \frac{m_{\mu}^{2} T^{4}}{m_{h}^{4} m_{h_{2}}^{4}} \int_{2 m_{\mu} / T}^{\infty} w^{8} K_{1}(w) \mathrm{d} w$,

which leads to the constraints on $v_{s}$ and $m_{h_{2}}$, as seen in Fig. 20. The invisible decay of $h_{1}$ requires a small $\theta$ or a low magnitude of $N$. In the resonance enhanced region $\left(2 m_{\mu}<m_{h_{2}}<4 \mathrm{GeV}\right)$, using the narrow resonance conditions of $\Gamma_{h_{2}} \ll m_{h_{2}}$, one obtains

$\langle\sigma v\rangle_{s_{N-1} s_{N-1} \rightarrow \mu^{+} \mu^{-}}=\frac{\lambda_{h s}^{2}}{256} \frac{m_{\mu}^{2} m_{h_{2}}^{6}}{T^{5} m_{h}^{4} \Gamma_{h_{2}}}\left(1-\frac{4 m_{\mu}^{2}}{m_{h_{2}}^{2}}\right)^{3 / 2} K_{1}\left(m_{h_{2}} / T\right)$,

which set lower bounds on the mixing angle of $\theta$; zsee Fig. 21. For a small $v_{s}$, the invisible decay of the $h_{1}$ set the upper limits on $\theta$ depending on the number of $N$ as shown in the left panel of Fig. 21. For the case of $m_{\mu}<m_{h_{2}}<2 m_{\mu}$, we have,

$$
\langle\sigma v\rangle_{s_{N-1} s_{N-1} \rightarrow \mu^{+} \mu^{-}}=\frac{\lambda_{h s}^{2}}{128 \pi} \frac{m_{\mu}^{2}}{m_{h}^{4}} \int_{2 m_{\mu} / T}^{\infty} w^{4} K_{1}(w) d w .
$$

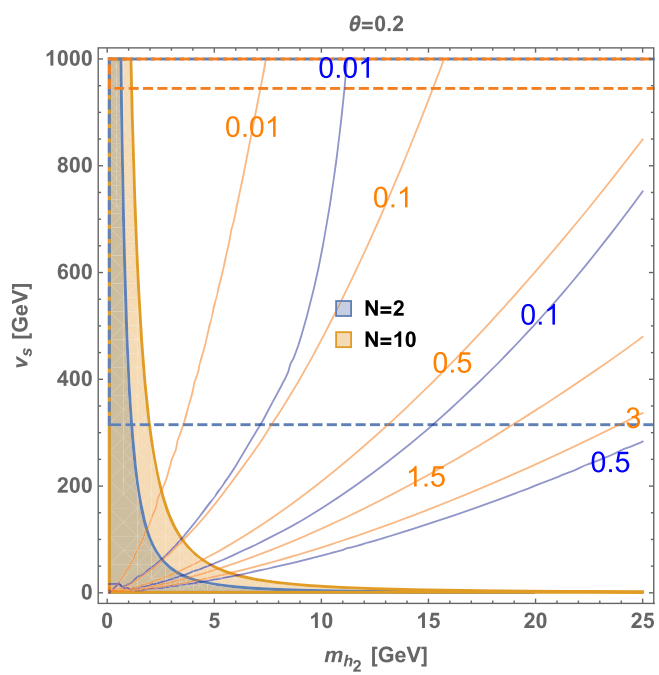

FIG. 20. Decouple conditions satisfied parameter regions in parameter spaces of $m_{h_{2}}$ and $v_{s}$, regions in the dashed square frame are allowed by the $B_{\text {inv }}<0.34$ [67]. The decay width of $h_{2}$ labeled on the blue and orange contours are shown in units of MeV. 

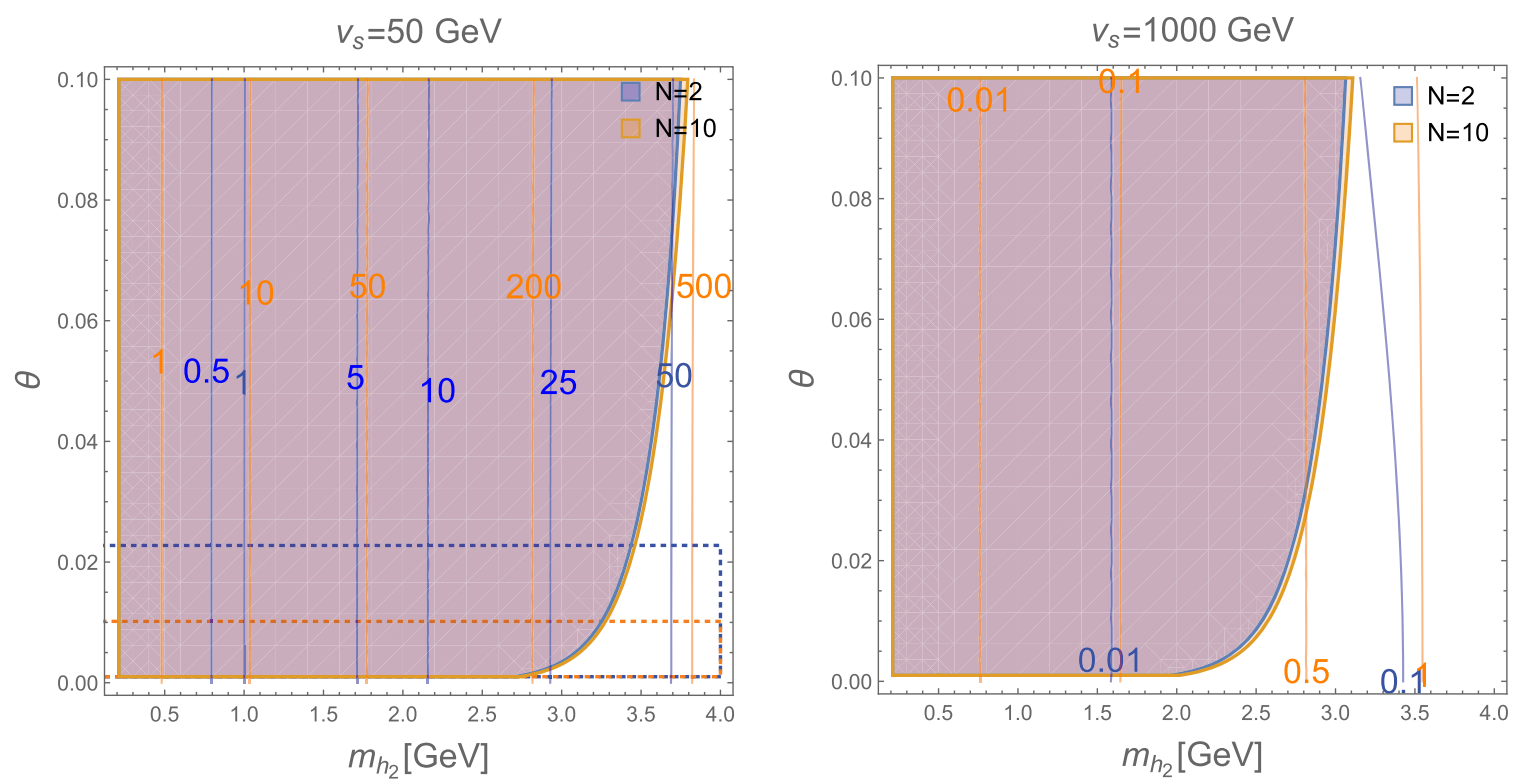

FIG. 21. Decouple conditions valid regions for $2 m_{\mu}<m_{h_{2}}<4 \mathrm{GeV}$, regions in the dashed square frame are allowed by the $B_{\text {inv }}<$ 0.34 [67]. The decay width of $h_{2}\left(\Gamma_{h_{2}}^{\text {tot }}\right)$ labeled on the contours is shown in units of $\mathrm{KeV}$.

Requiring that the Goldstone boson annihilation process contribute to the equivalent neutrino numbers, we obtain the bounds on the mixing angle and the $v_{s}$; see Fig. 22. A large $N$ requires a small $\theta$ to meet the decoupling conditions.

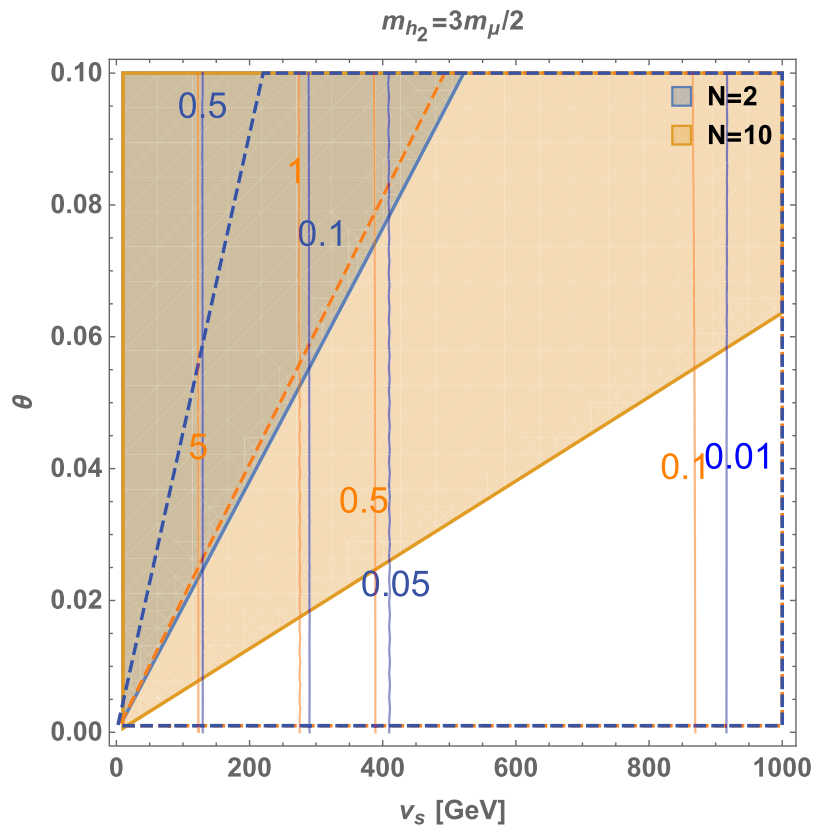

FIG. 22. Decouple conditions valid parameter regions for $m_{h_{2}}=3 m_{\mu} / 2$, regions in the dashed square frame are allowed by the $B_{\text {inv }}<0.34$ [67]. The decay width of $h_{2}\left(\Gamma_{h_{2}}^{\text {tot }}\right)$ labeled on the contours is shown in units of $\mathrm{KeV}$.

\section{CONCLUSIONS AND DISCUSSIONS}

In this work, we studied the possibility to realize the slowroll Higgs inflation and a SFOEWPT with the $N$-scalar extended SM. The condition of the successful inflation and a SFOEWPT set a stringent bound on the number of singlet scalars (or Goldstones) when the symmetry respected by the scalars is exact $O(N)$ symmetry (or the symmetry is broken to $O(N-1)$ ). The stability problem can easily be remedied up to the inflation scale with the assistance of the $N$ scalars that couple to the SM Higgs through the Higgs-portal interactions. Meanwhile, the perturbativity and the unitarity set severely upper bounds on the scalar quartic couplings with the increasing of the energy scale, especially with the increasing of the number of $N$. The Higgs inflation valid parameter regions of scalar quartic couplings diminish with the increase of the number $N$ mostly due to the perturbativity and the unitarity bounds.

The EWPOs severely constrain the parameters spaces of both $O(N)$ and $O(N \rightarrow N-1)$ scenarios. Further improvement on EWPOs constraints would restrict the $N$ more rigorous. For the $O(N)$ case, the number of $N$ validating the inflation is bounded to $N \leq 3$, which make both one-step and two-step SFOEWPT unreachable. Though all of the $N$ scalars can serve as WIMP DM candidates, no way to expect that the $N$-scalar WIMP DM can saturate the correct relic density here. This is because the masses of the $N$ scalars is of $\sim \mathcal{O}(1-10) \mathrm{TeV}$ scale considering the future $e^{+} e^{-}$colliders (such as ILC, FCC-ee, and CEPC) bounds. Here, the freeze out happens earlier than the EWPT process, thus the only relevant DM annihilation process is $S_{i} S_{i} \rightarrow h h$ with $m_{h}\left(T \geq T_{f s}\right) \sim 0$. 
When the $O(N)$ symmetry is spontaneously broken to $O(N \rightarrow N-1)$, one obtains $N-1$ Goldstones, and the one extra Higgs. Therefore, the invisible Higgs decay is very powerful to set the bound on the Goldstone number $N-1$ and the mixing angle $\theta$. With one moderate $\theta=0.2$ allowed by EWPO and Higgs precisions as well as invisible Higgs decay bounds set by LHC, we explore the possibility to realize Higgs inflation and a SFOEWPT through onestep and two-step types. In the parameter regions where a SFOEWPT can occur, the perturbativity problem appears at high scales might preclude the possibility to reach a successful inflation. In the parameter regions where one can obtain successful slow-roll Higgs inflation and a SFOEWPT, the triple Higgs couplings $\lambda_{h_{2} h_{1} h_{1}}$ and $\lambda_{h_{1} h_{1} h_{1}}$ increase with the increase of $\lambda_{h s}$. The decay widths of the two Higgses are not large enough to introduce significant interference effect in the resonant mass regions of Higgs pair productions. The future ILC, FCC-ee, and CEPC are more powerful to test the mixing angle and the number of Goldstones $N-1$ in comparison with the LHC, and can probe the inflation and SFOEWPT valid parameter regions. With the increase of the Goldstone number, we obtain a decrease parameter space of the scalar quartic couplings that can address successful inflation and a SFOEWPT. The gravitational wave signal search can tell the number of Goldstones for the one-step SFOEWPT, and the phase transition can be the two-step one if there is no relation with the number of Goldstones. We left the study to the future works. The dark radiation calculations indicate that Goldstones decouple from the thermal bath at mass ranges of small $m_{h_{2}}$.

One can expect the feature being explored in this work is general, the representation of a global or local symmetry respected by a hidden sector might be highly restricted if its contribution to the thermal potential and/or the inflationary scalar potential (directly or indirectly) is noticeable.

\section{ACKNOWLEDGMENTS}

L. G. B. thank Shufang Su, Zhen Liu, Wei Su, Jiayin Gu, Honglei Li for communications and discussions on future collider searches of Higgs physics. L. G. B. is grateful for helpful discussions on dimension-six operators and EWPT with Marcela Carena, Zhen Liu, and Marc Riembau. L. G. B. is also grateful for helpful discussions on the electroweak phase transition in the $O(N)$ scalars model from Toshinori Matsui. L. G. B. appreciates Hyun Min Lee for helpful discussion and comments on this paper. This work is supported by the National Natural Science Foundation of China under Grant No. 11605016 and No. 11847301, Basic Science Research Program through the National Research Foundation of Korea (NRF) funded by the Ministry of Education, Science and Technology (Grant No. NRF-2016R1A2B4008759), and Korea Research Fellowship Program through the National Research Foundation of Korea (NRF) funded by the Ministry of Science and ICT (2017H1D3A1A01014046).

\section{APPENDIX A: ONE-LOOP RENORMALIZATION GROUP EQUATIONS}

Following Ref. [73], beta functions for the $N$-singlet scalar model are given below,

$\beta_{g_{s}}=\frac{g_{s}^{3}}{(4 \pi)^{2}}(-7)+\frac{g_{s}^{3}}{(4 \pi)^{4}}\left(\frac{11}{6} g^{2}+\frac{9}{2} g^{2}-26 g_{s}^{2}-2 x_{h} y_{t}^{2}\right)$,

$\beta_{g}=\frac{g^{3}}{(4 \pi)^{2}}\left(-\frac{39-x_{h}}{12}\right)+\frac{g^{3}}{(4 \pi)^{4}}\left(\frac{3}{2} g^{2}+\frac{35}{6} g^{2}\right.$

$\left.+12 g_{s}^{2}-\frac{3}{2} x_{h} y_{t}^{2}\right)$

$\beta_{g^{\prime}}=\frac{g^{\prime 3}}{(4 \pi)^{2}}\left(\frac{81+x_{h}}{12}\right)+\frac{g^{\prime 3}}{(4 \pi)^{4}}\left(\frac{199}{18} g^{\prime 2}+\frac{9}{2} g^{2}\right.$

$\left.+\frac{44}{3} g_{s}^{2}-\frac{17}{6} x_{h} y_{t}^{2}\right)$,

$\begin{aligned} \beta_{\lambda_{h}=} & \frac{1}{(4 \pi)^{2}}\left(6\left(1+3 x_{h}^{2}\right) \lambda_{h}^{2}-6 y_{t}^{4}+\frac{3}{8}\left(2 g^{4}+\left(g^{2}+g^{\prime 2}\right)^{2}\right)\right. \\ & \left.+\lambda_{h} \gamma_{h}+\frac{N x_{s}^{2}}{2} \lambda_{h s}^{2}\right),\end{aligned}$

$\beta_{\lambda_{h s}}=\frac{\lambda_{s h}}{(4 \pi)^{2}}\left(6\left(x_{h}^{2}+1\right) \lambda_{h}+4 x_{h} x_{s} \lambda_{h s}+6 N x_{s}^{2} \lambda_{s}\right.$

$\left.+6 y_{t}^{2}-\frac{9}{2} g^{2}-\frac{3}{2} g^{\prime 2}\right)$,

$\beta_{\lambda_{s}}=\frac{1}{(4 \pi)^{2}}\left(18 N x_{s}^{2} \lambda_{s}^{2}+\frac{1}{2}\left(x_{h}^{2}+3\right) \lambda_{h s}^{2}\right)$,

$\beta_{y_{t}}=\frac{y_{t}}{(4 \pi)^{2}}\left[-\frac{9}{4} g^{2}-\frac{17}{12} g^{2}-8 g_{s}^{2}+\frac{23+4 x_{S}}{6} y_{t}^{2}\right]$.

with $\gamma_{h}=\left(-9 g^{2}-3 g^{\prime 2}+12 y_{t}^{2}\right), g, g^{\prime}$ and $y_{t}$ are the standard model $S U(2), U(1)$ and top-quark Yukawa couplings, and

$$
\begin{aligned}
& x_{h}=\frac{1+\xi_{h} h^{2} / M_{\mathrm{p}}^{2}}{1+\xi_{h} h^{2} / M_{\mathrm{p}}^{2}+6 \xi_{h}^{2} h^{2} / M_{\mathrm{p}}^{2}}, \\
& x_{S}=\frac{1+\xi_{S} S^{2} / M_{\mathrm{p}}^{2}}{1+\xi_{S} S^{2} / M_{\mathrm{p}}^{2}+6 \xi_{S}^{2} S^{2} / M_{\mathrm{p}}^{2}} .
\end{aligned}
$$

Following Refs. [73,76], The $\xi_{S}$ is set to zero at EW scale to ensure the kinematical mixing term canonical, and the $\xi_{h}$ is determined by CMB observations; see Eq. (44). The beta functions of $\xi_{h, S}$ can be found in Refs. $[73,76]$. The effects of $\xi_{S}$ here is negligible. The $\xi_{h}$ can lead to a tinny enhancement of the quartic couplings before the RG scale (here it is $h$ ) is comparable with the 
$M_{\mathrm{p}}$ since it's contribution to the beta functions is additive; see Eqs. (A4)-(A6).

\section{APPENDIX B: INGREDIENTS FOR ELECTROWEAK PHASE TRANSITIONS}

The tree level scalar potential for $O(N)$ scenario is obtained directly from Eq. (1),

$V_{0}(h, S)=-\frac{\mu^{2} h^{2}}{2}+\frac{\lambda h^{4}}{4}+\frac{\mu_{s}^{2} S^{2}}{2}+\frac{\lambda_{s} S^{4}}{4}+\frac{\lambda_{h s} h^{2} S^{2}}{4}$.

Here, we drop the subscript since all $\mathrm{N}$ directions are the same and we assume only one direction get VEV during the EWPT process. The "S" should be the "s" for the $O(N \rightarrow$ $N-1)$ scenario to indicate the possible symmetry breaking direction with other directions $s_{i}(i=1, \ldots, N-1)$ do not get VEV during the EWPT process. For the $O(N)$ scalar model, the one-loop Coleman-Weinberg potential for the scalar parts is given by

$$
\begin{aligned}
V_{C W}(h, S, N)= & \frac{1}{64 \pi^{2}}\left[m_{h}^{4}(h, S)\left(\log \frac{m_{h}^{2}(h, S)}{Q^{2}}-c_{i}\right)\right. \\
& +2 m_{G^{+}}^{4}(h, S)\left(\log \frac{m_{G^{+}}^{2}(h, S)}{Q^{2}}-3 / 2\right) \\
& +m_{G_{0}}^{4}(h, S)\left(\log \frac{m_{G_{0}}^{2}(h, S)}{Q^{2}}-3 / 2\right) \\
& \left.+N m_{S}^{4}(h, S)\left(\log \frac{m_{s}^{2}(h, S)}{Q^{2}}-3 / 2\right)\right] .
\end{aligned}
$$

If the $O(N)$ is broken to $O(N-1)$, we have

$$
\begin{aligned}
V_{C W}(h, s, N)= & \frac{1}{64 \pi^{2}}\left[m_{h_{1}}^{4}(h, s)\left(\log \frac{m_{h_{1}}^{2}(h, s)}{Q^{2}}-\frac{3}{2}\right)\right. \\
& +m_{h_{2}}^{4}(h, s)\left(\log \frac{m_{h_{2}}^{2}(h, s)}{Q^{2}}-\frac{3}{2}\right) \\
& +2 m_{G^{+}}^{4}(h, s)\left(\log \frac{m_{G^{+}}^{2}(h, s)}{Q^{2}}-\frac{3}{2}\right) \\
& +m_{G^{0}}^{4}(h, s)\left(\log \frac{m_{G^{0}}^{2}(h, s)}{Q^{2}}-\frac{3}{2}\right) \\
& \left.+(N-1) m_{s}^{4}(h, s)\left(\log \frac{m_{s}^{2}(h, s)}{Q^{2}}-\frac{3}{2}\right)\right] .
\end{aligned}
$$

For other gauge bosons contributions and fermions contributions we refer to Ref. [88]. The running scale $Q$ is chosen to be $Q=246.22 \mathrm{GeV}$ in the numerical analysis process. The field dependent masses are given as follows for both $O(N-1)$ and $O(N)$ cases (in this case one need do " $s$ " $\rightarrow$ " $S$ "),

$$
\begin{aligned}
m_{h s}(h, s) & =\lambda_{h s} h s \\
m_{h}^{2}(h, s) & =3 \lambda h^{2}-\mu^{2}+\frac{\lambda_{h s} s^{2}}{2}, \\
m_{s}^{2}(h, s) & =\frac{\lambda_{h s} h^{2}}{2}+\mu_{s}^{2}+3 \lambda_{s} s^{2}, \\
m_{G^{+}}^{2}(h, s) & =\lambda h^{2}-\mu^{2}+\frac{\lambda_{h s} s^{2}}{2}, \\
m_{G^{0}}^{2}(h, s) & =\lambda h^{2}-\mu^{2}+\frac{\lambda_{h s^{2}}}{2},
\end{aligned}
$$

and when $O(N)$ is broken to $O(N-1)$, we need to diagonalize the field-dependent mass matrix of $M=$ $\left(\left(m_{h}^{2}, m_{h s}\right),\left(m_{h s}, m_{s}^{2}\right)\right)$ to obtain the mass eigenvalue, i.e., $\left(m_{h_{1}}^{2}, m_{h_{2}}^{2}\right)$. The finite temperature corrections to the effective potential at one loop are given by [85]

$$
\begin{aligned}
V_{T}(h, S, N, T)= & \frac{T^{4}}{2 \pi^{2}}\left[J_{B}\left(\frac{m_{h}^{2}(h, S, N, T)}{T^{2}}\right)\right. \\
& +J_{B}\left(\frac{m_{G^{0}}^{2}(h, S, N, T)}{T^{2}}\right) \\
& +2 J_{B}\left(\frac{m_{G^{+}}^{2}(h, S, N, T)}{T^{2}}\right) \\
& \left.+N J_{B}\left(\frac{m_{S}^{2}(h, S, N, T)}{T^{2}}\right)\right]
\end{aligned}
$$

and

$$
\begin{aligned}
V_{T}(h, s, N, T)= & \frac{T^{4}}{2 \pi^{2}}\left[J_{B}\left(\frac{m_{h_{1}}^{2}(h, s, N, T)}{T^{2}}\right)\right. \\
& +J_{B}\left(\frac{m_{G^{0}}^{2}(h, s, N, T)}{T^{2}}\right) \\
& +2 J_{B}\left(\frac{m_{G^{+}}^{2}(h, s, N, T)}{T^{2}}\right) \\
& +J_{B}\left(\frac{m_{h_{2}}^{2}(h, s, N, T)}{T^{2}}\right) \\
& \left.+(N-1) J_{B}\left(\frac{m_{s}^{2}(h, s, N, T)}{T^{2}}\right)\right]
\end{aligned}
$$

for $O(N)$ and $O(N-1)$ scenarios, respectively, where the functions $J_{B}(y)$ are

$$
J_{B}(y)=\int_{0}^{\infty} d x x^{2} \ln \left[1-\exp \left(-\sqrt{x^{2}+y}\right)\right] .
$$

In addition, the above integral $J_{B}$ can be expressed as a sum of the second kind modified Bessel functions $K_{2}(x)$,

$$
J_{B}(y)=\lim _{N \rightarrow+\infty}-\sum_{l=1}^{N} \frac{(1)^{l} y}{l^{2}} K_{2}(\sqrt{y} l)
$$


The thermal masses/corrections are given by

$$
\begin{aligned}
m_{h}^{2}(h, S, N, T)= & m_{h_{2}}^{2}+\frac{1}{16} T^{2}\left(g_{1}^{2}+3 g_{2}^{2}+4 g_{t}^{2}\right) \\
& +T^{2}\left(\frac{\lambda}{2}+\frac{N \lambda_{h s}}{12}\right), \\
m_{G^{+}}^{2}(h, S, N, T)= & m_{G^{+}}^{2}+\frac{1}{16} T^{2}\left(g_{1}^{2}+3 g_{2}^{2}+4 g_{t}^{2}\right) \\
& +T^{2}\left(\frac{\lambda}{2}+\frac{N \lambda_{h s}}{12}\right), \\
m_{G^{0}}^{2}(h, S, N, T)= & m_{G^{0}}^{2}+\frac{1}{16} T^{2}\left(g_{1}^{2}+3 g_{2}^{2}+4 g_{t}^{2}\right) \\
& +T^{2}\left(\frac{\lambda}{2}+\frac{N \lambda_{h s}}{12}\right), \\
m_{S}^{2}(h, S, N, T)= & m_{S}^{2}+T^{2}\left(\frac{(N+2) \lambda_{s}}{4}+\frac{\lambda_{h s}}{3}\right),
\end{aligned}
$$

for the $O(N)$ case, and for the $O(N \rightarrow N-1)$ case one needs to replace the " $S$ " by "s" and replace the thermal mass of the Higgs fields by

$$
\begin{aligned}
m_{h_{1}}^{2}(h, N, T)= & m_{h_{1}}^{2}+\frac{1}{16} T^{2}\left(g_{1}^{2}+3 g_{2}^{2}+4 g_{t}^{2}\right) \\
& +T^{2}\left(\frac{\lambda}{2}+\frac{N \lambda_{h s}}{12}\right), \\
m_{h_{2}}^{2}(h, N, T)= & m_{h_{2}}^{2}+\frac{1}{16} T^{2}\left(g_{1}^{2}+3 g_{2}^{2}+4 g_{t}^{2}\right) \\
& +T^{2}\left(\frac{(N+2) \lambda_{s}}{4}+\frac{\lambda_{h s}}{3}\right),
\end{aligned}
$$

Last but not least, the resummation of daisy diagrams is also crucial for the evaluation of $v_{C}$ and $T_{C}$ with the finite temperature effective potential $[103,104]$, which is given by

$$
\begin{aligned}
V_{\text {daisy }}(h, S, N, T)= & \frac{T}{12}\left[\left(m_{h}^{3}-m_{h}^{3}(h, S, N, T)\right)\right. \\
& +\left(m_{G^{0}}^{3}-m_{G^{0}}^{3}(h, S, N, T)\right. \\
& \left.+2\left(m_{G^{+}}^{3}-m_{G^{+}}^{3}(h, S, N, T)\right)\right] \\
& \left.+N\left(m_{S}^{3}-m_{S}^{3}(h, S, N, T)\right)\right]
\end{aligned}
$$

and

$$
\begin{aligned}
V_{\text {daisy }}(h, s, N, T)= & \frac{T}{12}\left[\left(m_{h_{1}}^{3}-m_{h_{1}}^{3}(h, s, N, T)\right)\right. \\
& +3\left(m_{G^{0}}^{3}-m_{G^{0}}^{3}(h, s, N, T)\right) \\
& +\left(m_{h_{2}}^{3}-m_{h_{2}}^{3}(h, s, N, T)\right) \\
& \left.+(N-1)\left(m_{s}^{3}-m_{s}^{3}(h, s, N, T)\right)\right],
\end{aligned}
$$

for $O(N)$ and $O(N \rightarrow N-1)$ cases. Here, again, we list only the contributions of scalar contributions for $V_{T}$ and $V_{\text {daisy }}$, the other particle field contributions are the same as the SM; see Refs. [88,104]. It should be noted that the counter terms can keep the VEVs of the potential from shifts caused by the $V_{C W}$; we add that parts follow Ref. [88].

\section{APPENDIX C: DARK MATTER CALCULATION APPROACH OF $O(N)$ CASE}

For SM Higgs pair final states, the annihilation cross section is given by

$$
\begin{aligned}
\left\langle\sigma v_{\text {rel }}\right\rangle_{h h}= & \frac{\lambda_{h s}^{2}}{64 \pi m_{s}^{2}}\left[1+\frac{3 m_{h}^{2}}{\left(4 m_{s}^{2}-m_{h}^{2}\right)}+\frac{2 \lambda_{h s} v^{2}}{\left(m_{h}^{2}-2 m_{s}^{2}\right)}\right]^{2} \\
& \times\left(1-\frac{m_{h}^{2}}{m_{s}^{2}}\right)^{1 / 2}
\end{aligned}
$$

the cross section for gauge boson final states is

$$
\begin{aligned}
\left\langle\sigma v_{\text {rel }}\right\rangle_{W W}= & 2\left[1+\frac{1}{2}\left(1-\frac{2 m_{s}^{2}}{m_{W}^{2}}\right)^{2}\right]\left(1-\frac{m_{W}^{2}}{m_{s}^{2}}\right)^{1 / 2} \\
& \times \frac{\lambda_{h s}^{2} m_{W}^{4}}{8 \pi m_{S}^{2}\left(\left(4 m_{s}^{2}-m_{h}^{2}\right)^{2}+m_{h}^{2} \Gamma_{h}^{2}\right)}, \\
\left\langle\sigma v_{\text {rel }}\right\rangle_{Z Z}= & 2\left[1+\frac{1}{2}\left(1-\frac{2 m_{S}^{2}}{m_{Z}^{2}}\right)^{2}\right]\left(1-\frac{m_{Z}^{2}}{m_{S}^{2}}\right)^{1 / 2} \\
& \times \frac{\lambda_{h s}^{2} m_{Z}^{4}}{16 \pi m_{S}^{2}\left(\left(4 m_{S}^{2}-m_{h}^{2}\right)^{2}+m_{h}^{2} \Gamma_{h}^{2}\right)}
\end{aligned}
$$

and the fermion pair final states cross section is given by $\left\langle\sigma v_{\text {rel }}\right\rangle_{\bar{f} f}=\frac{m_{W}^{2}}{\pi g^{2}} \frac{\lambda_{f}^{2} \lambda_{h s}^{2}}{\left(4 m_{S}^{2}-m_{h}^{2}\right)^{2}+m_{h}^{2} \Gamma_{h}^{2}}\left(1-\frac{m_{f}^{2}}{m_{S}^{2}}\right)^{3 / 2}$

The formula of the spin-independent cross section is given by [98]

$$
\sigma_{S I}^{S}=\lambda_{h s}^{2} \frac{f_{N}^{2}}{4 \pi}\left(\frac{m_{N} m_{S}}{\left(m_{N}+m_{S}\right)}\right)^{2} \frac{m_{N}^{2}}{m_{H}^{4} m_{S}^{2}}
$$

where $m_{N}=0.946 \mathrm{GeV}$ is the neutron mass and $m_{H}=$ $126 \mathrm{GeV}$ is the SM-Higgs mass. The strengths of the hadronic matrix elements, $f_{N}=0.35$. The dark matter direct detection constrains the dark matter masses and the quartic Higgs-DM couplings after taking into account the rescale effects, assuming the evaluated dark matter relic density does not oversaturate the DM relic abundance,

$$
\sigma_{S I}=\sigma_{S I}^{S_{i}} \times \sum_{i=1, \ldots, N} \frac{\Omega^{S_{i}} h^{2}}{\Omega_{\mathrm{DM}} h^{2}}
$$


[1] A. H. Guth, Phys. Rev. D 23, 347 (1981).

[2] A. A. Starobinsky, Phys. Lett. 91B, 99 (1980).

[3] A. D. Linde, Phys. Lett. 108B, 389 (1982).

[4] P. A. R. Ade et al. (Planck Collaboration), Astron. Astrophys. 594, A20 (2016).

[5] F. L. Bezrukov and M. Shaposhnikov, Phys. Lett. B 659, 703 (2008).

[6] A. O. Barvinsky, A. Y. Kamenshchik, and A. A. Starobinsky, J. Cosmol. Astropart. Phys. 11 (2008) 021.

[7] Z. Z. Xianyu and H. J. He, J. Cosmol. Astropart. Phys. 10 (2014) 083.

[8] J. Ellis, H. J. He, and Z. Z. Xianyu, J. Cosmol. Astropart. Phys. 08 (2016) 068.

[9] J. Ellis, H. J. He, and Z. Z. Xianyu, Phys. Rev. D 91, 021302 (2015).

[10] For other nonminimally coupled models, see Refs. [11-13].

[11] L. Marzola and A. Racioppi, J. Cosmol. Astropart. Phys. 10 (2016) 010.

[12] M. Artymowski and A. Racioppi, J. Cosmol. Astropart. Phys. 04 (2017) 007.

[13] A. Racioppi, Phys. Rev. D 97, 123514 (2018).

[14] G. Aad et al. (ATLAS Collaboration), Phys. Lett. B 716, 1 (2012).

[15] S. Chatrchyan et al. (CMS Collaboration), Phys. Lett. B 716, 30 (2012).

[16] C. P. Burgess, H. M. Lee, and M. Trott, J. High Energy Phys. 09 (2009) 103.

[17] J. L. F. Barbon and J. R. Espinosa, Phys. Rev. D 79, 081302 (2009).

[18] A. O. Barvinsky, A. Y. Kamenshchik, C. Kiefer, A. A. Starobinsky, and C. F. Steinwachs, Eur. Phys. J. C 72, 2219 (2012).

[19] F. Bezrukov, A. Magnin, M. Shaposhnikov, and S. Sibiryakov, J. High Energy Phys. 01 (2011) 016.

[20] C. P. Burgess, H. M. Lee, and M. Trott, J. High Energy Phys. 07 (2010) 007.

[21] M. P. Hertzberg, J. High Energy Phys. 11 (2010) 023.

[22] X. Calmet and R. Casadio, Phys. Lett. B 734, 17 (2014).

[23] C. P. Burgess, S. P. Patil, and M. Trott, J. High Energy Phys. 06 (2014) 010.

[24] F. Bezrukov, J. Rubio, and M. Shaposhnikov, Phys. Rev. D 92, 083512 (2015).

[25] J. Fumagalli and M. Postma, J. High Energy Phys. 05 (2016) 049.

[26] V. M. Enckell, K. Enqvist, and S. Nurmi, J. Cosmol. Astropart. Phys. 07 (2016) 047.

[27] For a resolution of the controversy see Ref. [28]. Ref. [7] gave the first natural minimal SM Higgs inflation without invoking nonminimal coupling and any new particles below UV-scale under asymptotical safe approach.

[28] J. Ren, Z. Z. Xianyu, and H. J. He, J. Cosmol. Astropart. Phys. 06 (2014) 032.

[29] D. E. Morrissey and M. J. Ramsey-Musolf, New J. Phys. 14, 125003 (2012).

[30] M. D’Onofrio, K. Rummukainen, and A. Tranberg, Phys. Rev. Lett. 113, 141602 (2014).

[31] N. Arkani-Hamed, T. Han, M. Mangano, and L. T. Wang, Phys. Rep. 652, 1 (2016).

[32] J. R. Espinosa, T. Konstandin, and F. Riva, Nucl. Phys. B854, 592 (2012).
[33] S. Profumo, M. J. Ramsey-Musolf, C. L. Wainwright, and P. Winslow, Phys. Rev. D 91, 035018 (2015).

[34] P. H. Ghorbani, J. High Energy Phys. 08 (2017) 058.

[35] P. H. Ghorbani, Phys. Rev. D 98, 115016 (2018).

[36] K. Ghorbani and P. H. Ghorbani, arXiv:1804.05798.

[37] A. Alves, T. Ghosh, H. K. Guo, K. Sinha, and D. Vagie, arXiv:1812.09333.

[38] A. Alves, T. Ghosh, H. K. Guo, and K. Sinha, J. High Energy Phys. 12 (2018) 070.

[39] M. Jiang, L. Bian, W. Huang, and J. Shu, Phys. Rev. D 93, 065032 (2016).

[40] C. W. Chiang, M. J. Ramsey-Musolf, and E. Senaha, Phys. Rev. D 97, 015005 (2018).

[41] R. N. Lerner and J. McDonald, Phys. Rev. D 83, 123522 (2011).

[42] G. Ballesteros, J. Redondo, A. Ringwald, and C. Tamarit, Phys. Rev. Lett. 118, 071802 (2017).

[43] H. J. He and Z. Z. Xianyu, J. Cosmol. Astropart. Phys. 10 (2014) 019.

[44] For the Higgs inflation with no-scale SUSY GUT, we refer to Refs. $[8,9]$.

[45] W. Cheng and L. Bian, Phys. Rev. D 98, 023524 (2018).

[46] D. Curtin, P. Meade, and C. T. Yu, J. High Energy Phys. 11 (2014) 127.

[47] T. Tenkanen, K. Tuominen, and V. Vaskonen, J. Cosmol. Astropart. Phys. 09 (2016) 037.

[48] K. Enqvist, S. Nurmi, T. Tenkanen, and K. Tuominen, J. Cosmol. Astropart. Phys. 08 (2014) 035.

[49] J. R. Espinosa and M. Quiros, Phys. Rev. D 76, 076004 (2007).

[50] J. R. Espinosa, T. Konstandin, J. M. No, and M. Quiros, Phys. Rev. D 78, 123528 (2008).

[51] M. Kakizaki, S. Kanemura, and T. Matsui, Phys. Rev. D 92, 115007 (2015).

[52] K. Hashino, M. Kakizaki, S. Kanemura, and T. Matsui, Phys. Rev. D 94, 015005 (2016).

[53] L. Bian, R. Ding, and B. Zhu, Phys. Lett. B 728, 105 (2014).

[54] L. Bian, T. Li, J. Shu, and X. C. Wang, J. High Energy Phys. 03 (2015) 126.

[55] K. Endo and Y. Sumino, J. High Energy Phys. 05 (2015) 030.

[56] The WIMP DM situation in the classical scale invariant $N$-scalars model with $O(N)$ symmetry [55] are ruled out for $N>4$ even for rather large quartic coupling [57].

[57] K. Endo and K. Ishiwata, Phys. Lett. B 749, 583 (2015).

[58] The breaking of $O(N)$ can happen in any direction, means we can have any of $s_{i}$ with $i=1,2, \ldots, N$ obtain VEV, here we assume $O(N)$ breaks in $s_{N}$ direction.

[59] C. Grojean, G. Servant, and J. D. Wells, Phys. Rev. D 71, 036001 (2005).

[60] B. Henning, X. Lu, and H. Murayama, arXiv:1404.1058.

[61] B. Henning, X. Lu, and H. Murayama, J. High Energy Phys. 01 (2016) 023.

[62] N. Craig, C. Englert, and M. McCullough, Phys. Rev. Lett. 111, 121803 (2013).

[63] M. Baak, J. Cuth, J. Haller, A. Hoecker, R. Kogler, K. Mnig, M. Schott, and J. Stelzer (Gfitter Group Collaboration), Eur. Phys. J. C 74, 3046 (2014).

[64] Indeed, if one want the heavy Higgs take part in the EWPT process, it cannot be highly decoupled. 
[65] V. Barger, P. Langacker, M. McCaskey, M. J. RamseyMusolf, and G. Shaughnessy, Phys. Rev. D 77, 035005 (2008).

[66] M. Carena, Z. Liu, and M. Riembau, Phys. Rev. D 97, 095032 (2018).

[67] G. Aad et al. (ATLAS and CMS Collaborations), J. High Energy Phys. 08 (2016) 045.

[68] J. Gu, H. Li, Z. Liu, S. Su, and W. Su, J. High Energy Phys. 12 (2017) 153.

[69] K. Fujii et al., arXiv:1506.05992.

[70] M. Bicer et al. (TLEP Design Study Working Group), J. High Energy Phys. 01 (2014) 164.

[71] D. d'Enterria, arXiv:1602.05043.

[72] CEPC-SPPC Study Group, Reports No. IHEP-CEPC-DR2015-01, No. IHEP-TH-2015-01, and No. IHEP-EP-201501, 2015, http://inspirehep.net/record/1395734.

[73] R. N. Lerner and J. McDonald, Phys. Rev. D 80, 123507 (2009).

[74] E. Elizalde and S. D. Odintsov, Phys. Lett. B 321, 199 (1994).

[75] E. Elizalde, S. D. Odintsov, E. O. Pozdeeva, and S. Y. Vernov, Phys. Rev. D 90, 084001 (2014).

[76] A. Aravind, M. Xiao, and J. H. Yu, Phys. Rev. D 93, 123513 (2016); 96, 069901(E) (2017).

[77] T. E. Clark, B. Liu, S. T. Love, and T. ter Veldhuis, Phys. Rev. D 80, 075019 (2009).

[78] We note that Refs. [79,80] drop the kinematic mixing terms due to suppress of the largeness of the non-minimal coupling combination, and the Higgs inflation conditions there are satisfied in our case.

[79] O. Lebedev and H. M. Lee, Eur. Phys. J. C 71, 1821 (2011).

[80] O. Lebedev, Eur. Phys. J. C 72, 2058 (2012).

[81] L. Kofman, A. D. Linde, and A. A. Starobinsky, Phys. Rev. Lett. 73, 3195 (1994).

[82] F. Bezrukov, D. Gorbunov, and M. Shaposhnikov, J. Cosmol. Astropart. Phys. 06 (2009) 029.
[83] J. Garcia-Bellido, D. G. Figueroa, and J. Rubio, Phys. Rev. D 79, 063531 (2009).

[84] F. L. Bezrukov, arXiv:0805.2236.

[85] L. Dolan and R. Jackiw, Phys. Rev. D 9, 3320 (1974).

[86] H. H. Patel and M. J. Ramsey-Musolf, J. High Energy Phys. 07 (2011) 029.

[87] P. B. Arnold and O. Espinosa, Phys. Rev. D 47, 3546 (1993); 50, 6662(E) (1994).

[88] J. Bernon, L. Bian, and Y. Jiang, J. High Energy Phys. 05 (2018) 151.

[89] P. Huang, A. Joglekar, B. Li, and C. E. M. Wagner, Phys. Rev. D 93, 055049 (2016).

[90] C. Delaunay, C. Grojean, and J. D. Wells, J. High Energy Phys. 04 (2008) 029.

[91] J. M. Cline and K. Kainulainen, J. Cosmol. Astropart. Phys. 01 (2013) 012.

[92] L. Bian and X. Liu, arXiv:1811.03279.

[93] L. Bian and Y. L. Tang, J. High Energy Phys. 12 (2018) 006.

[94] E. K. Akhmedov, Z. G. Berezhiani, R. N. Mohapatra, and G. Senjanovic, Phys. Lett. B 299, 90 (1993).

[95] S. R. Coleman, Nucl. Phys. B310, 643 (1988).

[96] O. Catà, A. Ibarra, and S. Ingenhütt, J. Cosmol. Astropart. Phys. 11 (2017) 044.

[97] E. W. Kolb and K. A. Olive, Phys. Rev. D 33, 1202 (1986); 34, 2531(E) (1986).

[98] J. M. Cline, K. Kainulainen, P. Scott, and C. Weniger, Phys. Rev. D 88, 055025 (2013); 92, 039906 (2015).

[99] G. Steigman, Phys. Rev. D 87, 103517 (2013).

[100] G. Steigman, arXiv:1310.6259.

[101] P. A. R. Ade et al. (Planck Collaboration), Astron. Astrophys. 571, A16 (2014).

[102] C. Garcia-Cely, A. Ibarra, and E. Molinaro, J. Cosmol. Astropart. Phys. 11 (2013) 061.

[103] G. W. Anderson and L. J. Hall, Phys. Rev. D 45, 2685 (1992).

[104] M. E. Carrington, Phys. Rev. D 45, 2933 (1992). 